\title{
Plasma environment of Titan: a 3-D hybrid simulation study
}

\author{
S. Simon ${ }^{1}$, A. Bößwetter ${ }^{1}$, T. Bagdonat ${ }^{1}$, U. Motschmann ${ }^{1,2}$, and K.-H. Glassmeier ${ }^{3}$ \\ ${ }^{1}$ Institute for Theoretical Physics, TU Braunschweig, Germany \\ ${ }^{2}$ Institute for Planetary Research, DLR, Berlin, Germany \\ ${ }^{3}$ Institute for Geophysics and Extraterrestrial Physics, TU Braunschweig, Germany
}

Received: 4 January 2006 - Accepted: 23 February 2006 - Published: 19 May 2006

\begin{abstract}
Titan possesses a dense atmosphere, consisting mainly of molecular nitrogen. Titan's orbit is located within the Saturnian magnetosphere most of the time, where the corotating plasma flow is super-Alfvénic, yet subsonic and submagnetosonic. Since Titan does not possess a significant intrinsic magnetic field, the incident plasma interacts directly with the atmosphere and ionosphere. Due to the characteristic length scales of the interaction region being comparable to the ion gyroradii in the vicinity of Titan, magnetohydrodynamic models can only offer a rough description of Titan's interaction with the corotating magnetospheric plasma flow. For this reason, Titan's plasma environment has been studied by using a 3-D hybrid simulation code, treating the electrons as a massless, charge-neutralizing fluid, whereas a completely kinetic approach is used to cover ion dynamics. The calculations are performed on a curvilinear simulation grid which is adapted to the spherical geometry of the obstacle. In the model, Titan's dayside ionosphere is mainly generated by solar UV radiation; hence, the local ion production rate depends on the solar zenith angle. Because the Titan interaction features the possibility of having the densest ionosphere located on a face not aligned with the ram flow of the magnetospheric plasma, a variety of different scenarios can be studied. The simulations show the formation of a strong magnetic draping pattern and an extended pick-up region, being highly asymmetric with respect to the direction of the convective electric field. In general, the mechanism giving rise to these structures exhibits similarities to the interaction of the ionospheres of Mars and Venus with the supersonic solar wind. The simulation results are in agreement with data from recent Cassini flybys.
\end{abstract}

Keywords. Magnetospheric physics (Magnetosphereionosphere interactions; Planetary magnetospheres) - Space plasma physics (Kinetic and MHD theory)

Correspondence to: S. Simon

(sven.simon@tu-bs.de)

\section{Introduction}

With its extended neutral atmosphere, Titan orbits Saturn at a distance of 20.3 Saturn radii and an orbital period of 15.95 days. Titan's orbit is located inside Saturn's outer magnetosphere for average solar wind conditions, since under these circumstances the stand-off distance of the bow shock has been observed to vary typically between 23.6 and $31.5 \mathrm{Sa}-$ turn radii (Ledvina et al., 2004a). The magnetosphere is populated by neutral atoms and plasma from several potential sources. On the one hand, Saturn's atmosphere and rings as well as the icy satellites can contribute to the plasma population inside the magnetosphere. On the other hand, plasma from Titan's ionosphere and solar wind particles are also part of the plasma population. Theoretical predictions suggest that the plasma at least partially corotates with the planet (Saur et al., 2004). Due to Titan's orbital period being considerable larger than Saturn's rotational period $(10.7 \mathrm{~h})$, Titan is permanently embedded into a flow of magnetized plasma with a relative velocity around $120 \mathrm{~km} / \mathrm{s}$. The data that have been collected during the Voyager 1 flyby (Ness et al., 1982) as well as the magnetic field signature observed during the first Cassini encounter (Backes et al., 2005) suggest that Titan does not possess a significant intrinsic magnetic field. Thus, Titan's atmosphere and ionosphere interact directly with the Saturnian magnetospheric plasma in a similar way as Venus and Mars interact with the solar wind. However, the Titan interaction possesses several unique features.

The stand-off distance of both Saturn's bow shock and magnetopause will decrease at times of high solar wind dynamic pressure. Under these circumstances, the bow shock might be located at a distance of 20 Saturn radii (Schardt et al., 1984), and therefore, Titan might be able to leave the magnetosphere in the subsolar region of its orbital period and hence, the moon interacts with the unshocked solar wind. Besides, the ionospheric properties on the side of Titan that faces the oncoming plasma flow vary with Saturnian local time. Especially, if solar UV radiation is assumed to

Published by Copernicus GmbH on behalf of the European Geosciences Union. 


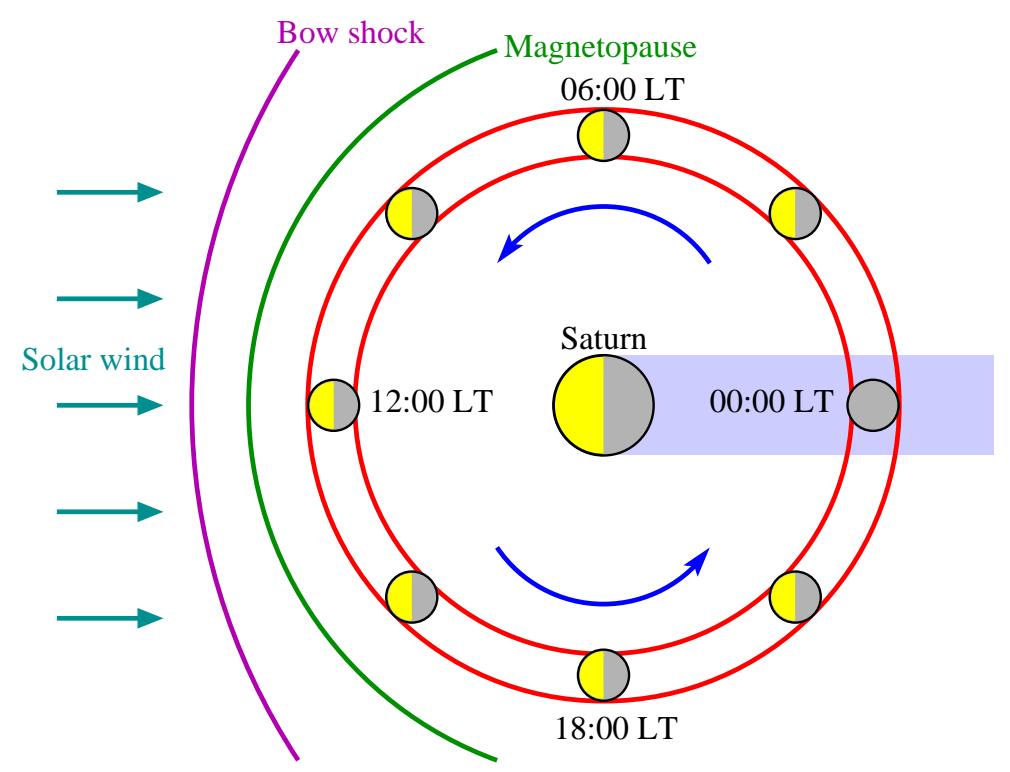

Fig. 1. Titan's orbit around Saturn. The abbreviation "LT" in the sketch denotes the Saturnian local time. Titan orbits Saturn at a distance of 20.3 Saturn radii with an orbital period of 15.95 days. Since this period is significantly longer than Saturn's rotational period, Titan's atmosphere is permanently exposed to a flow of corotating magnetospheric plasma. The blue arrows denote Titan's rotational direction as well as the direction of the corotating plasma flow. The plasma flow velocity with respect to Titan is of the order of $120 \mathrm{~km} / \mathrm{s}$. As can be seen from the figure, the Titan situation features the possibility of having the dayside ionosphere located on a face not aligned with the ram flow of the Saturnian magnetospheric plasma, leading to a variety of different interaction scenarios. Titan's orbit is located inside Saturn's magnetosphere for average solar wind conditions when the stand-off distance of the bow shock is of the order of 24 Saturn radii. However, when Saturn's magnetosphere is compressed due to high solar wind dynamic pressure, Titan might leave the magnetosphere in the subsolar region of its orbital period. Under these circumstances, the ionosphere can interact directly with the supersonic solar wind. Such a compression of Saturn's magnetosphere was detected during the Pioneer 11 flyby (Schardt et al., 1984).

be the major ionization source, the region of maximum ion production is not necessarily located in the hemisphere being exposed to the upstream corotating plasma flow. This situation is illustrated in Fig. 1.

Various simulation studies on the subject of plasma interaction with Titan have already been carried out. Most of these models use the magnetohydrodynamic approximation and hence are only capable of covering the global characteristics of the interaction region. Keller et al. (1994) used a one-dimensional multispecies magnetohydrodynamic model to study the dynamics of hydrocarbon ions in the ramside ionosphere of Titan. The sources of ionization and the electron temperature profiles incorporated into this model depend on the relative locations of Titan, Saturn and the sun. An analogeous one-dimensional model for the wakeside ionosphere has been developed by Keller and Cravens (1994). In this approach, electron impact ionization of Titan's atmosphere by radially streaming Saturnian magnetospheric electrons was assumed to be the only source of ion production, whereas the ramside model included both photoionization and electron impact.

Cravens et al. (1998) studied the global features of Titan's plasma environment by using a 2-D, multifluid magnetohydrodynamic model which maintains high spatial resolution in Titan's ionosphere by means of a grid possessing cylindrical geometry and non-uniform radial grid spacing. Three different ion species have been incorporated into these model calculations. In agreement with data from the Voyager 1 flyby, this model shows that no bow shock arises in front of the obstacle. Besides, the authors suggest that at the flanks of the wake region, Titan has a noticable effect on Saturn's magnetospheric plasma flow only for radial distances below 10 Titan radii. However, since this model cannot cover the draping of the ambient magnetic field around the obstacle, the formation of a magnetotail in the downstream region does not occur in the simulation results. In order to be capable of describing these aspects of Titan's plasma environment, the model has been extended to three spatial dimensions by Ledvina and Cravens (1998) who analyzed the global characteristics of the interaction region for two different cases: On the one hand, the simulation parameters have been chosen in accordance to data from the Voyager 1 flyby. On the other hand, the upstream plasma flow was assumed to be both supersonic and super-Alfvénic. This scenario might be of importance for those times when Titan might find itself in the solar wind. The nominal distance of 
Saturn's bow shock at the subsolar point is larger than Titan's orbital distance. Variations in the solar wind can lead to a compression of Staturn's magnetosphere so that the orbit of Titan may be found in the magnetosheath or even out in the solar wind. The model results indicate that for upstream plasma conditions similar to those observed during the Voyager 1 flyby, Titan possesses a narrow plasma wake with a diameter of only $1-2$ Titan radii $\left(R_{T}=2575 \mathrm{~km}\right)$. With respect to the size of the obstacle, such a tail is considerable smaller than the induced magnetotails of Venus or Mars (Kivelson and Russell, 1983; Luhmann et al., 1991; Verigin et al., 1984). In agreement with the results of Ness et al. (1982), the authors show that - as long as Titan is located inside the Saturnian magnetosphere - the interaction does not only result in the formation of a strong magnetic draping pattern, but gives also rise to a set of Alfvén wings. On the other hand, if the upstream plasma flow is supersonic, the simulation results show the formation of a bow shock denoting a sharp decrease of plasma velocity.

Another 3-D magnetohydrodynamic model covering the general large-scale features of the interaction between Saturn's magnetospheric plasma and Titan's upper atmosphere has been developed by Kabin et al. (1999). The effects of a conducting ionosphere, exospheric mass loading and ionneutral charge exchange are taken into consideration. Although Titan certainly does not possess a strong intrinsic magnetic field (Ness et al., 1982; Backes et al., 2005), the authors try to explain several details of the observed plasma signatures in the vicinity of Titan by assuming the existence of a small intrinsic magnetic dipole field. In a companion paper, Kabin et al. (2000b) use the results of a numerical MHD model to compare the magnetic field topology in Titan's wake to an idealized picture of field lines draping around a conductive, non-magnetic obstacle. Another 3-D resistive MHD study emphasizing the importance of mass loading effects in the vicinity of Titan has been carried out by Kopp and Ip (2001). The authors suggest the existence of a significant asymmetry in the structure of the mass-loading patters between the Saturn-facing hemisphere and the antiSaturn hemisphere of Titan. The results of this study are in general agreement with Voyager 1 measurements. In contrast to all other simulation studies mentioned above, both the MHD models of Kabin et al. (2000a) and Kopp and Ip (2001) assume a non-uniform ion production profile, i.e. the ion production rate is depending on the solar zenith angle.

A similar 3-D MHD simulation model has been developed by Nagy et al. (2001) who take into consideration the effects of exospheric mass-loading as well as major chemical reactions and ion-neutral collisions. Three different ion species are incorporated into the model calculations. In preparation for the first close flyby of Titan by Cassini, Ma et al. (2004) developed a 3-D multispecies global MHD model. By using a spherical structured simulation grid, the authors were able to achieve an extremely good altitude resolution of about $36 \mathrm{~km}$ in the ionospheric region of Titan. In this approach,
Titan's atmosphere and ionosphere have been approximated by 10 neutral and 7 ion species, each of them represented by a continuity equation. The simulation results do not only predict the magnetic field signatures for the first Cassini flyby, but are also in reasonably good agreement with the data collected during the Voyager 1 encounter. Recently, Backes et al. (2005) used a 3-D resistive MHD model to interprete the magnetic field signature that has been observed in the vicinity of Titan during the first Cassini encounter on 26 October 2004.

The results of the MHD simulations reproduce the overall features of the interaction region quite well. However, due to the ion gyroradius not being small compared to Titan, it is clear that ion kinetic effects also play a role for the structure of the interaction region and that the MHD approximation is not strictly applicable to the case of Titan. Besides, most MHD codes include only one ion species and do not carry the Hall term. Since such a model is not capable of displaying diamagnetic effects, it must return a symmetric solution. Such a model is incapable of covering the effects arising from the ion's finite gyroradius. However, due to the fluid description of the plasma, these codes have proven adequate to accommodate the complex chemical processes occuring in the ionosphere. Moreover, they can cover larger regions where the dynamics and asymmetries due to the Hall effect are only of minor importance. In contrast to this, large-scale hybrid or full-particle simulations require extensive computing capacity.

Nevertheless, the need for a self-consistent model, such as a quasi-neutral hybrid model, that takes into account finite gyroradius effects, has already been emphasized by Ledvina et al. (2004b) who calculated the trajectories of several thousand ions in the vicinity of Titan by using the field parameters obtained from a 3-D MHD model of Titan's plasma interaction. A similar combination of magnetohydrodynamic and test particle simulations has been realized by Ledvina et al. (2000) to make predictions for the Cassini orbiter particle experiments. Besides, by incorporating test particles into the results of self-consistent MHD simulations, the velocity space distributions of several ion species in the vicinity of Titan have been studied by Ledvina et al. (2005). The importance of finite gyroradius effects has also been illustrated by Luhmann (1996) who analyzed the motion of individual ions in Titan's plasma wake. However, neither of these models is capable of offering a completely self-consistent description of Titan's plasma environment.

The first who used a self-consistent hybrid model to study Titan's plasma environment were Brecht et al. (2000), refering to the case that Titan encounters a supersonic co-rotating Saturnian magnetospheric plasma. The simulation results indicate that Titan's magnetotail exhibits an asymmetry with respect to the direction of the convective electric field and hence, demonstrated the importance of ion kinetic effects. Nevertheless, due to the magnetospheric plasma temperature being neglected, this model is only capable of offer- 
ing a rough approximation to the real Titan situation. Further hybrid simulations based on the same assumption have been carried out by Ledvina et al. (2004a). Kallio et al. (2004) were the first who applied the hybrid approximation to the case that the upstream plasma flow is super-Alfvénic, yet subsonic and submagnetosonic. However, this model does not take into consideration the plasma's finite electron pressure, i.e. the electron temperature is put to zero in the momentum equation describing the dynamics of the electron fluid. Since the pressure term has already proven to be of extreme importance in hybrid simulation studies of solar wind interaction with comets (Bagdonat, 2004) and Mars (Bößwetter et al., 2004), incorporating this term into the model and analyzing its effects on the global structure of the interaction region is a primary goal of this work.

The purpose of our work is to analyse Titan's plasma environment as a function of Saturnian local time. Emphasis is placed on identifying the mechanism giving rise to the plasma signatures in the vicinity of the satellite. To the author's knowledge, this is the first hybrid simulation study to offer a systematic discussion of the changes in Titan's plasma environment during the satellite's orbital period. The data collected during Cassini's $T_{A}$ flyby on 25 October 2004 show that Titan's plasma wake exhibits a significantly asymmetric structure (Wahlund et al., 2005). Based on our simulation results, the mechanism leading to the formation of these asymmetries can be explained by analyzing the Lorentz forces acting on individual ions. It will be demonstarted that both the convective electric field and the electron pressure gradient are of major importance for understanding Titan's plasma interaction. The paper is arranged as follows: The second section deals with the specifications of the 3-D hybrid code as well as the ionosphere model. In the third chapter, Titan's highly variable plasma environment will be analyzed for different upstream conditions:

- At first, the general characteristics of the interaction region will be discussed for the case of a supersonic, supermagnetosonic and super-Alfvénic upstream flow. On the one hand, the results provided by this scenario will help to validate the simulation model since it qualitatively resembles the interaction of Mars with the solar wind. The Martian plasma environment has been studied in detail by Bößwetter et al. (2004). On the other hand, it will be demonstrated that the interaction mechanism in this case is of major importance for understanding Titan's plasma environment when the moon is located inside Saturn's magnetosphere.

- When Titan is located inside Saturn's magnetosphere, the upstream plasma flow is super-Alfvénic, yet subsonic and submagnetosonic (Wolf and Neubauer, 1982). As can be seen from Fig. 1, Titan's dayside is exposed to the corotating plasma flow at 18:00 Saturnian local time. This situation will be considered in Sect. 3.2.
- At 06:00 Saturnian local time, the corotating magnetospheric plasma interacts with Titan's nightside ionosphere. For this case, a comparative discussion of the interaction region's major features will be given in Sect. 3.3.

- Wolf and Neubauer (1982) have stated that the plasma flow interacting with Titan's ionosphere will be subsonic, submagnetosonic and sub-Alfvénic for Titan being located in Saturn's tail region. Besides, due to Titan's atmosphere not being exposed to solar UV radiation in this case, the satellite's ionospheric structure will differ significantly from the other three cases under consideration. This situation will be discussed in Sect. 3.4.

The simulation model does not yet take into account seasonal variations of Titan's plasma environment, i.e. we refer to the situation at the equinoxes of Saturn's orbital period.

The paper concludes with a summary and an outlook to future projects.

\section{Model description}

\subsection{Basic equations of the hybrid model}

In the hybrid model, the electrons are treated as a massless, charge-neutralizing fluid, whereas a completely kinetic description is retained to cover ion dynamics. The simulation code which has been applied to Titan's plasma environment is a modified version of the 3-D hybrid code developed by Bagdonat and Motschmann (2001; 2002a; 2002b), being capable of realizing the spatial discretization by means of an arbitrary curvilinear simulation gird. This code has already been applied successfully to the Martian plasma environment (Bößwetter et al., 2004).

As stated, the ions are treated as individual particles. Hence, their dynamics are described by the equations of motion

$\frac{\mathrm{d} \underline{x}_{s}}{\mathrm{~d} t}=\underline{v}_{s} \quad$ and $\quad \frac{\mathrm{d} \underline{v}_{s}}{\mathrm{~d} t}=\frac{q_{s}}{m_{s}}\left\{\underline{E}+\underline{v}_{s} \times \underline{B}\right\} \quad$,

being equivalent to the characteristics of the Vlasov equation for the respective ion species. The quantities $\underline{x}_{s}$ and $\underline{v}_{s}$ denote the position and the velocity of an ion of species 's', respectively, whereas $\underline{E}$ and $\underline{B}$ are the electromagnetic field quantities. On the other hand, the description of electron dynamics is based on the momentum equation

$0=n_{e} m_{e} \frac{\mathrm{d} \underline{u}_{e}}{\mathrm{~d} t}=-e n_{e}\left(\underline{E}+\underline{u}_{e} \times \underline{B}\right)-\nabla P_{e}$,

which yields an explicit expression for the electric field:

$\underline{E}=-\underline{u}_{e} \times \underline{B}-\frac{1}{e n_{e}} \nabla P_{e}$.

The quantities $n_{e}, \underline{u}_{e}$ and $P_{e}$ are the electron fluid's mean density, velocity and pressure, respectively. However, since 


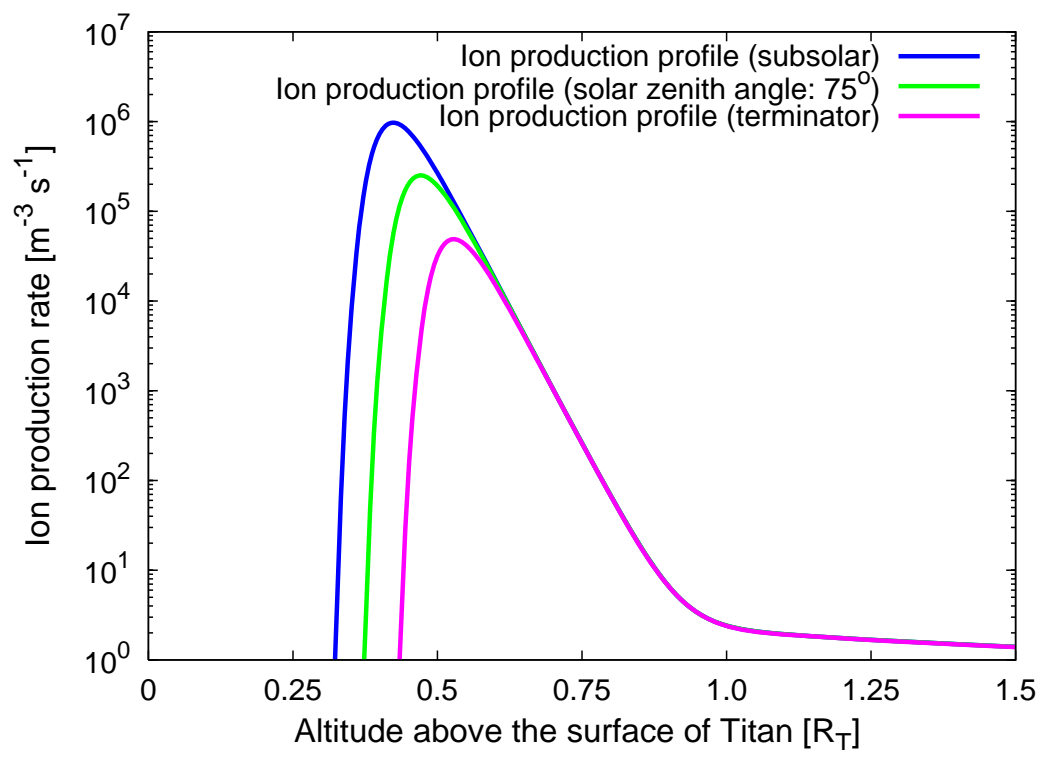

Fig. 2. Production of molecular nitrogen as a function of altitude above the surface. The figure displays the production rate $q_{v}$ at the subsolar point (green line), at a solar zenith angle of $\chi_{\nu}=75^{\circ}$ (green line) and at the terminator (magenta line). As can also be seen in Fig. 3 , the altitude of the production maximum increases when moving from the subsolar point to the terminator. In contrast to this, the value of maximum production diminishes for large solar zenith angles.

the electron temperature in the corotating magnetospheric plasma differs significantly form the temperature in Titan's ionosphere (Roboz and Nagy, 1994; Nagy and Cravens, 1998), two different electron populations, denoted by the subscripts $\mathrm{m}$ (magnetospheric) and $\mathrm{i}$ (ionospheric), have been incorporated into the simulation model:

$0=-e n_{e, m}\left(\underline{E}+\underline{u}_{e, m} \times \underline{B}\right)-\nabla P_{e, m}$

$0=-e n_{e, i}\left(\underline{E}+\underline{u}_{e, i} \times \underline{B}\right)-\nabla P_{e, i}$.

By introducing the cumulative electron density

$n_{e} \equiv n_{e, m}+n_{e, i} \quad$,

and the mean electron velocity

$\underline{u}_{e}=\frac{n_{e, m}}{n_{e}} \underline{u}_{e, m}+\frac{n_{e, i}}{n_{e}} \underline{u}_{e, i}$,

Eqs. (4) and (5) yield the following expression for the electric field:

$\underline{E}=-\underline{u}_{e} \times \underline{B}-\frac{\nabla P_{e, m}+\nabla P_{e, i}}{e n_{e}}$.

Both electron populations are assumed to be adiabatic, i.e.

$P_{e, m} \propto \beta_{e, m} n_{e, m}^{\kappa} \quad$ and $\quad P_{e, i} \propto \beta_{e, i} n_{e, i}^{\kappa} \quad$.

Due to the thermodynamic coupling in a collision-free plasma being realized in only two spatial dimensions, a value of $\kappa=2$ has been chosen for the adiabatic exponent. The set of hybrid equations is completed by Faraday's law,

$\nabla \times \underline{E}=-\frac{\partial \underline{B}}{\partial t}$,

describing the time evolution of the magnetic field.

\subsection{Ionosphere model}

At the beginning of each simulation run, Titan does not possess an ionosphere. Ions of ionospheric origin are continuously produced while the simulation proceeds, i.e. a certain number of these particles is inserted into the simulation domain during each time step. This situation evolves until a quasi-stationary state is reached.

Titan's atmosphere is modeled as a spherical symmetric gas cloud surrounding the obstacle and consisting of molecular nitrogen. Only this single ionospheric species is taken into account. For the simulations described in this work, a neutral density profile according to

$$
\begin{aligned}
& n_{n}(r)= \\
& =n_{1} \exp \left(\frac{r_{1}-r}{H_{1}}\right)+n_{2} \exp \left(\frac{r_{2}-r}{H_{2}}\right)+n_{3} \frac{r_{3}}{r}
\end{aligned}
$$

has been used. The profile contains three reference densities $\left(n_{1}, n_{2}\right.$ and $\left.n_{3}\right)$ as well as three reference heights $\left(r_{1}, r_{2}\right.$ and $r_{3}$ ), allowing to adjust the profile to the results of ionosphere model calculations. The scale lengths $H_{1}$ and $H_{2}$ allow the inclusion of data from ion temperature measurements. The decrease $\mathrm{d} I$ of solar $\mathrm{UV}$ intensity due to absorption processes can be obtained from

$\frac{\mathrm{d} I}{I}=\sigma_{v} n_{n}(r) \frac{\mathrm{d} r}{\cos \chi_{v}}$, 
Table 1. Input parameters for the ion production profile according to Eq. (14). The radius of Titan is $R_{T}=2575 \mathrm{~km}$.

\begin{tabular}{lll}
\hline Parameter & Symbol & Numerical value \\
\hline Neutral profile (first term) & $n_{1}$ & $1.0 \cdot 10^{18} \mathrm{~m}^{-3}$ \\
& $r_{1}$ & $700 \mathrm{~km}$ \\
& $H_{1}$ & $90 \mathrm{~km}$ \\
\hline Neutral profile (second term) & $n_{2}$ & $1.0 \cdot 10^{12} \mathrm{~m}^{-3}$ \\
& $r_{2}$ & $1700 \mathrm{~km}$ \\
\hline Neutral profile (third term) & $H_{2}$ & $120 \mathrm{~km}$ \\
\hline Absorption cross-section & $n_{3}$ & $1.0 \cdot 10^{10} \mathrm{~m}^{-3}$ \\
\hline Photoionization efficiency & $r_{3}$ & $2700 \mathrm{~km}^{-22}$ \\
\hline Photoionization frequency & $\sigma_{v}$ & $8.37 \cdot 10^{-22} \mathrm{~m}^{2}$ \\
\hline Lower boundary of atmosphere & $\kappa_{v}$ & 1 \\
\hline Upper boundary of atmosphere & $v=\sigma_{\nu} I_{\infty}$ & $0.2 \cdot 10^{-9} \mathrm{~s}^{-1}$ \\
\hline Total ion production & $r_{\min }$ & $R_{T}+1000 \mathrm{~km}$ \\
\hline
\end{tabular}

where $\sigma$ is the absorption cross-section and $\chi_{v}$ denotes the solar zenith angle (Bauer, 1973; Baumjohann and Treumann, 1999; Bauer and Lammer, 2004). Using Eq. (11) yields

$I(r)=I_{\infty} \exp \left[-\frac{\sigma_{v}}{\cos \chi_{v}} \int_{r}^{r_{\max }} n_{n}(\tilde{r}) \mathrm{d} \tilde{r}\right]$,

where $r_{\max }$ is the radius of a shell denoting the upper boundary of the ionosphere (Baumjohann and Treumann, 1999). For the simulations discussed in this work, a value of $r_{\max }=3 R_{T}$ has been chosen. As to be seen in Figs. 2 and 3, this assumption is uncritical. $I_{\infty}$ is the solar flux at the top of the atmosphere. The remaining integral can be solved analytically:

$$
\begin{aligned}
& \int_{r}^{r_{\max }} n_{n}(\tilde{r}) \mathrm{d} \tilde{r} \\
& =n_{1} H_{1} \exp \left(\frac{r_{1}-r}{H_{1}}\right)+n_{2} H_{2} \exp \left(\frac{r_{2}-r}{H_{2}}\right) \\
& +n_{3} r_{3} \ln \left(\frac{r_{\max }}{r}\right)-\left[n_{1} H_{1} \exp \left(\frac{r_{1}-r_{\max }}{H_{1}}\right)\right. \\
& \left.+n_{2} H_{2} \exp \left(\frac{r_{2}-r_{\max }}{H_{2}}\right)\right] .
\end{aligned}
$$

As the outer radius $r_{\text {max }}$ is considerable larger than the reference heights $r_{1}$ and $r_{2}$, the terms in Eq. (13) not depending on $r$ can be neglected. Hence, the ion production function $q_{v}$ is given by

$$
\begin{aligned}
& q_{v}(r)=\kappa_{v} \sigma_{v} n_{n}(r) I_{\infty} . \\
& \exp \left\{-\frac{\sigma_{v}}{\cos \chi_{v}}\left[n_{1} H_{1} \exp \left(\frac{r_{1}-r}{H_{1}}\right)+\right.\right.
\end{aligned}
$$

$$
\left.\left.+n_{2} H_{2} \exp \left(\frac{r_{2}-r}{H_{2}}\right)+n_{3} r_{3} \ln \left(\frac{r_{\max }}{r}\right)\right]\right\},
$$

where $\kappa_{v}$ is the photoionization efficiency. To sum up the major idea, in the simulation model, the ionosphere is assumed to be a spherical shell with the upper radius $r_{\max }$ and a lower boundary located at $r_{\min }=R_{T}+1000 \mathrm{~km}$, i.e at an altitude of $1000 \mathrm{~km}$ above the surface. In other words, the atmosphere is assumed to begin at an altidude of $r_{\min }$ above the surface and is cut off at the height $r_{\max }$. Thus, the total ion production $Q$ in this shell, i.e. the number of ions globally produced per second, can be obtained from

$$
Q=\int_{r_{\min }}^{r_{\max }} \int_{0}^{\pi} \int_{0}^{2 \pi} \mathrm{d} r \mathrm{~d} \theta \mathrm{d} \phi q_{\nu}(r) r^{2} \sin (\theta)
$$

Since the argument of the exponential term in Eq. (14) depends on $\left(\cos \chi_{\nu}\right)^{-1}$, this expression is not adequate for modelling the production rate near the terminator line. Furthermore, photoionization does not occur at the nightside of Ti$\tan$. For the simulations described in this work, the production for $\chi_{\nu} \approx 90^{\circ}$ as well as the nightside production have been approximated in a quite simple way: For both solar zenith angles $\chi_{v}>87^{\circ}$ and the nightside of the obstacle, the production is assumed to be independent of the solar zenith angle. The production function in these regions has been set to

$q_{v}\left(r, \chi_{v}\right)=q_{v}\left(r, \chi_{v}=87^{\circ}=\mathrm{const}\right) \quad$.

A value of $\chi_{v}=87^{\circ}$ instead of $\chi_{\nu}=90^{\circ}$ has been chosen since the $1 / \cos \chi_{v}$ term in the argument of the exponential function in Eq. (14) diverges at the terminator line. It is clear that 


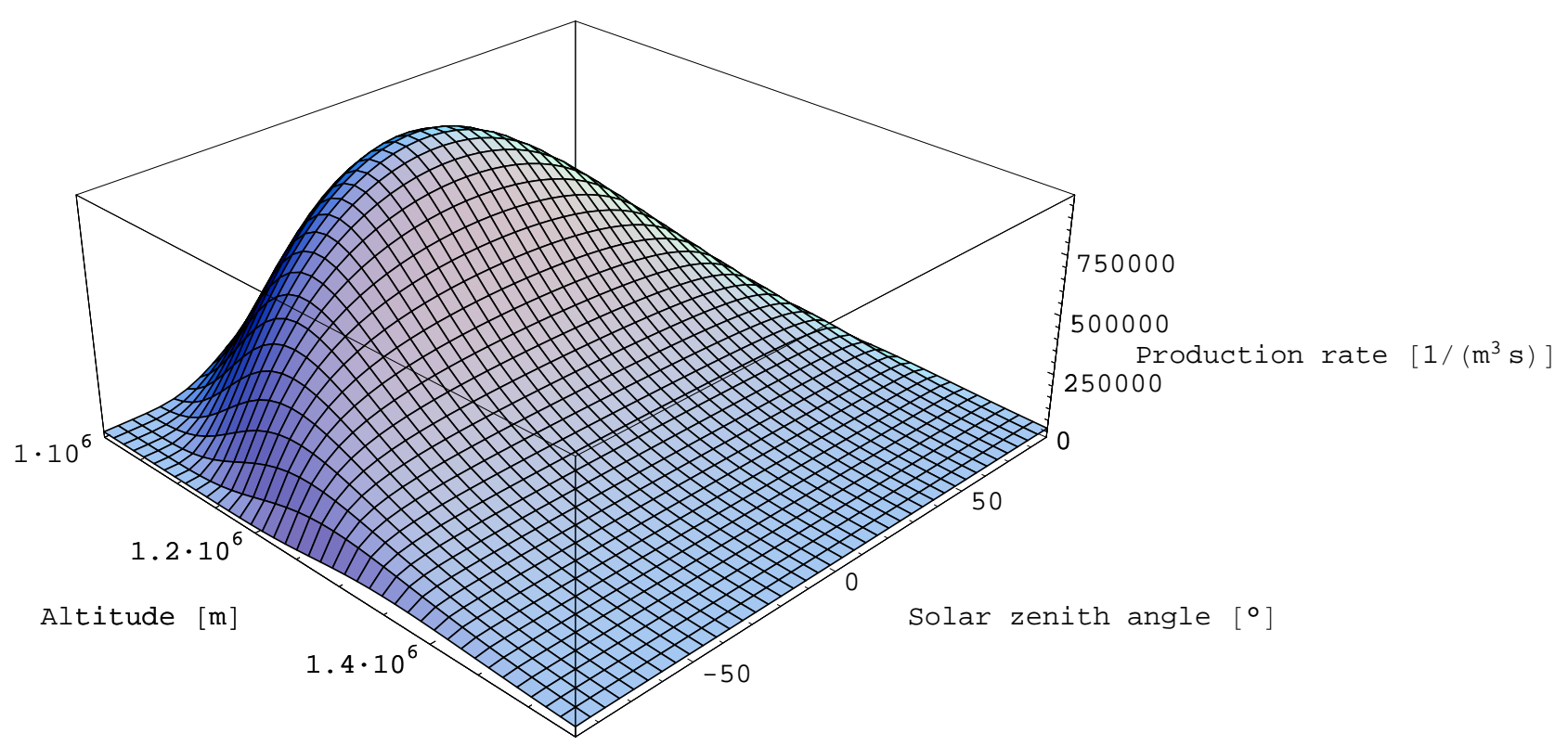

Fig. 3. Ion production rate of molecular nitrogen as a function of altitude and solar zenith angle. The production function is given by Eq. (14), the numerical values used to generate the profile can be found in Table 1.

this is only a rough approximation to the ionization processes occuring at the nightside.

The values for the free parameters in Eq. (14) are listed in Table 1 and have been chosen to match the profiles discussed by Nagy and Cravens (1998). The resulting ion production rates are shown in Figs. 2 and 3, respectively. When Titan is located in the Saturnian wake at 00:00 LT, solar ultraviolet radiation cannot be considered an ionization source. Since the electron impact processes becoming predominant in this situation cannot be covered by our simulation model, an approximative approach has been chosen to mimic the ionosphere at 00:00 LT. Our approach is based on the assumption that the ionosphere at 00:00 LT is significantly less pronounced than in situations where Titan is exposed to solar radiation. For the 00:00 LT situation, a weak and isotropic production rate has been incorporated into the model, i.e. the production function does not depend on the solar zenith angle. It is clear that this approach only allows a qualitative analysis of the interaction region.

\subsection{Boundary conditions}

The lower boundary of Titan's atmosphere is represented by an absorptive sphere, i.e. any particle hitting the surface of the obstacle is removed from the simulation scenario. However, in order to avoid strong electric fields arising from the resulting pressure gradient, the mean ion density at the grid points inside the obstacle has to be kept on a spatially constant, non-vanishing value. Due to the ionospheric denisty increasing continuously as the simulation proceeds, a time-dependent function has to be chosen for the inner density. Nevertheless, the model does not include a boundary condition for the electromagnetic fields, i.e. the field equations are solved outside as well as inside the obstacle. Although these boundary conditions have already proven to be adequate for hybrid simulations of planetary plasma environments (Bößwetter et al., 2004), they allow the magnetic field to diffuse into the obstacle's interior. A complete supression of these numerical diffusion effects seems to be impossible.

\subsection{Simulation parameters}

Titan's plasma environment has been analyzed by using a cubic simulation domain with a spatial extension of $15 R_{T}$ in each direction. In all simulations, the coordinate system's $x$ axis is parallel to the direction of the solar UV radiation, i.e. pointing at the dayside of Titan. For the simulation runs discussed in this work, the undisturbed upstream magnetic field is orientated antiparallel to the $z$ axis:

$\underline{B}_{0}=(0,0,-5 \mathrm{nT})$.

Yoyager 1 determined that Saturn's magnetospheric plasma in the general vicinity of Titan is composed of $N^{+}$and $H^{+}$with number densities of $0.1 \mathrm{~cm}^{-3}$ and $0.2 \mathrm{~cm}^{-3}$ (Ness et al., 1982; Hartle et al., 1982). In the simulations, we try to mimic this situation by using a magnetospheric plasma flow that consists of a single ion species of mass $m=(1 \cdot 1.0+2 \cdot 14.0) / 3 \mathrm{amu}=9.7 \mathrm{amu}$ and particle density $n_{0}=(0.1+0.2) \mathrm{cm}^{-3}=0.3 \mathrm{~cm}^{-3}$. An analogeous approach has shown to be successful in previous magnetohydrodynamic studies (Kabin et al., 1999) as well as in a hybrid 
model developed by Kallio et al. (2004). Particles are continuously injected into the simulation domain from all faces of the simulation box except for the wall being located in the wake region behind the obstacle. The upstream plasma velocity is given by $u_{0}=120 \mathrm{~km} / \mathrm{s}$. As the Alfvén velocity is

$v_{A, 0}=\frac{B_{0}}{\sqrt{9.7 \mu_{0} n_{0} m_{p}}}=64 \mathrm{~km} / \mathrm{s}$,

the Alfvénic Mach number of the upstream plasma flow is $M_{A}=u_{0} / v_{A, 0}=1.87$, i.e. the flow is super-Alfvénic. In the runs concerning the situation at 06:00 and 18:00 Saturnian local time, the plasma temperatures have been chosen in accordance to the parameters from the Voyager 1 flyby: The magnetospheric ion temperature has been set to $k_{B} T=2003 \mathrm{eV}$, yielding a plasma beta of $\beta=9.69$. A value of $k_{B} T=200 \mathrm{eV}$ has been chosen for the magnetospheric electron temperature; the corresponding plasma beta is $\beta=0.97$. Hence, the sonic and magnetosonic Mach numbers of the upstream plasma flow are

$M_{S}=0.57 \quad$ (sonic Mach number)

and

$M_{M S}=0.55 \quad$ (magnetosonic Mach number)

In agreement with the ionospheric temperature profiles proposed by Roboz and Nagy (1994), the electron temperature in the ionosphere has been approximated by $k_{B} T=0.17 \mathrm{eV}$, whereas the nitrogen ions of ionospheric origin are assumed to be cold.

The case of Titan being exposed to a supersonic plasma flow has been studied in numerous simulation models, but spacecraft data for this situation are not available. For the simulation study presented in this paper, the case of supersonic flow will provide a reference to understand the basic principle of the interaction mechanism when Titan is located inside Saturn's magnetosphere, but however, this situation is only of minor quantitative relevance. Since in this case, we are only interested in the general characteristics of the interaction region, the supersonic simulation scenario has been created from the 18:00 LT run in a very simple way: The Mach numbers have been altered to $M_{A}=1.87$, $M_{S}=2.9$ and $M_{M S}=1.6$, whereas all other plasma parameters (including the composition) are the same as for the innermagnetospheric runs. Thus, this simulation will offer a general impression of how Titan's plasma environment is structured in the supersonic case, but it is only of minor quantitative significance. In the present study, emphasis is placed on analyzing the general features of the interaction region as a function of the upstream Mach numbers, and not on a precise quantitative reproduction of Titan's plasma environment when the satellite is located in the solar wind. Although the parameters of the supersonic scenario differ from the real situation, they allow a direct comparison of the relevant mechanism to the subsonic case since only a minor change of the simulation parameters has been performed. Especially, the characteristic length and time scales (ion gyroradii and gyrofrequencies) in the upstream plasma are the same for both simulations. The set of Mach numbers has been chosen in agreement with magnetohydrodynamic simulations carried out by Ledvina et al. (2004a).

In another simulation run, Titan is assumed to be located in the Saturnian wake. Wolf and Neubauer (1982) have stated that under these circumstances the impinging plasma flow is sub-Alfvénic as well as subsonic and submagnetosonic. Due to lack of concrete data for this situation, the Mach numbers of the upstream plasma flow have been chosen in accordance with Ledvina et al. (2004a) who have studied the plasma environment of Titan being located in Saturn's wake by means of a magnetohydrodynamic code. The values of the three Mach numbers are $M_{A}=0.77$ (Alfvénic), $M_{S}=0.29$ (sonic) and $M_{M S}=0.27$ (magnetosonic).

The spatial discretization is realized by using a grid with $90 \times 90 \times 90$ cells. The center of the cubic simulation domain coincides with the center of Titan; the box possesses and extension of $\pm 7.5 R_{T}$ in each spatial direction. The simulations have been carried out sufficiently long to reach a quasi stationary-state. The total simulation time corresponds to a duration in which the undisturbed plasma flow would cross the entire simulation domain 28-30 times. Such a long duration is mandatory due to the extremely slow motion of the ionospheric particles in the tail region beyond the planet. In order to prevent local gradients from compromising numerical stability, a 27 point smoothing procedure has been applied.

\subsection{Simulation grid}

The simulations have been carried out on a curvilinear grid, taking into account the spherical structure of the obstacle and its ionosphere. This grid is derived from an equidistant Cartesian grid by modifying the radial distance of the grid points from the center. The following discussion refers to a cubic simulation domain of volume $L^{3}$ and the same number $N$ of grid points in each spatial direction. The quantity $L$ as well as all other lengths occuring in this section are dimensionless, they are given in units of Titan's radius $R_{T}$. The grid points of the Cartesian reference grid will be refered to as

$\underline{r}_{i j k}^{C}=\left(x_{i}^{C}, y_{j}^{C}, z_{k}^{C}\right)$,

where the three components are given by

$$
\begin{aligned}
x_{i}^{C} & =\left(\frac{i}{N}-\frac{1}{2}\right) L \quad, \quad i=0,1,2, \ldots, N \quad ; \\
y_{j}^{C} & =\left(\frac{j}{N}-\frac{1}{2}\right) L \quad, \quad j=0,1,2, \ldots, N \quad ; \\
z_{k}^{C} & =\left(\frac{k}{N}-\frac{1}{2}\right) L \quad, \quad k=0,1,2, \ldots, N .
\end{aligned}
$$




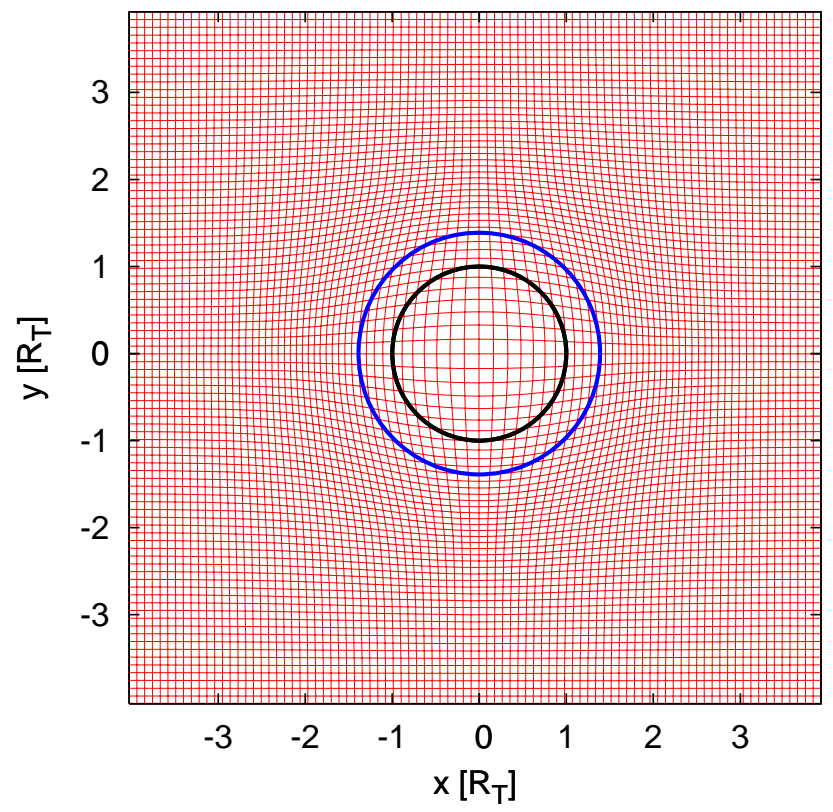

Fig. 4. Curvilinear fisheye simulation grid. In order to avoid the formation of numerical artifacts in the structure of the heavy ion cloud, a curvilinear simulation grid taking account for the spherical symmetry of the obstacle has been used. The inner black circle in the sketch denotes the position of Titan's surface, whereas the outer one marks the inner boundary of the ionosphere. The fisheye grid is obtained from an equidistant Cartesian grid by means of a coordinate transformation according to Eq. (29). The basic idea is to modify the radial distance of the Cartesian grid points from the center by using a scaling function $f(r)$. The function $f(r)$ that is utilized to generate the fisheye grid resembles the Fermi-Dirac distribution function. As can be seen from the figure, the major feature of this grid is a region of curved grid lines in the vicinity of the obstacle, whereas with increasing distance to the center of the simulation domain, the grid slightly transforms into a rectangular one. The scaling function $f(r)$ includes three free parameters $(\lambda, \mu$ and $v$ ) determining both the curvature of the gird and the location of the region with maximum curvature.

Thus, the radial distance of the grid point $(i, j, k)$ from the center can be written as

$r_{i j k}^{C}=\left|r_{i j k}^{C}\right|=\sqrt{\left(x_{i}^{C}\right)^{2}+\left(y_{j}^{C}\right)+\left(z_{k}^{C}\right)^{2}}$,

so that the position of the respective grid point is

$$
\underline{r}_{i j k}^{C}=\underbrace{\frac{\underline{r}_{i j k}^{C}}{r_{i j k}^{C}}}_{=\underline{e}} r_{i j k}^{C}=r_{i j k}^{C} \underline{e} .
$$

The vector $\underline{e}$ is a unit vector pointing from the origin of the Cartesian system to the point $\underline{r}_{i j k}^{C}$. In order to generate the

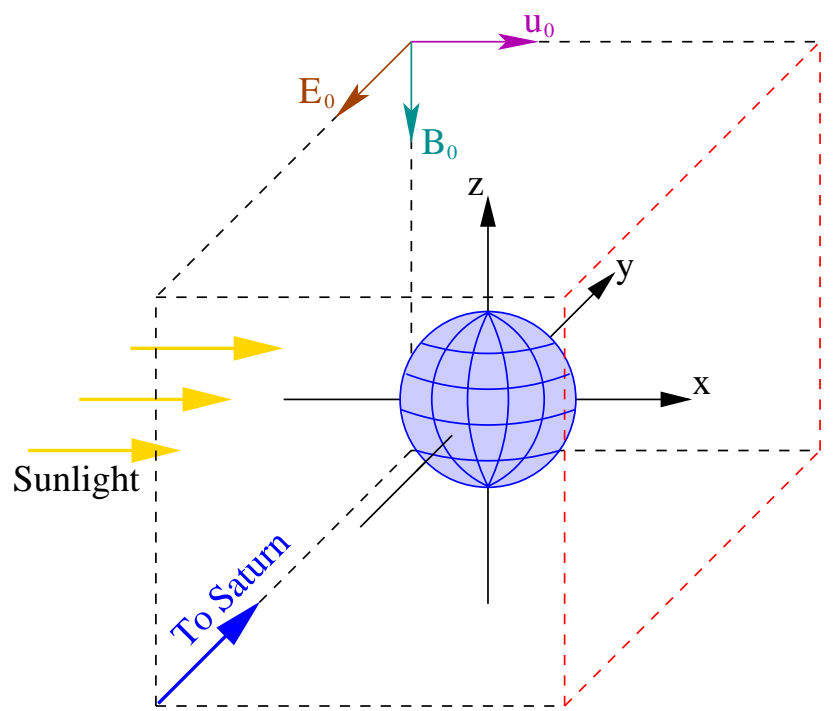

Fig. 5. Titan in subsonic and supersonic flow - simulation geometry. The interaction of Titan's ionosphere with the superAlfvénic, yet subsonic and submagnetosonic Saturnian magnetospheric plasma flow has been studied by using a cubic simulation domain with a length of $15 R_{T}$ in each direction. The center of the obstacle coincides with the origin of the Cartesian coordinate system shown in the sketch. The undisturbed plasma flow velocity points in $(+x)$ direction; the homogeneous background magnetic field is orientated in $(-z)$ direction. Thus, the convective electric field is antiparallel to the $(+y)$ axis and points away from Saturn. The dayside of the obstacle is located in the $(x<0)$ half space, i.e. the obstacle's terminator plane coincides with the $(y, z)$ plane. The boundary that is located in the wake region is denoted by red lines. The simulation geometry for Titan being exposed to a superAlfvénic, supersonic and supermagnetosonic plasma flow is exactly the same as the one shown in the sketch. Only the numerical values of the upstream plasma betas differ from the first simulation.

curvilinear fisheye grid, the radial distance is modified according to the transformation

$r_{i j k}^{C} \rightarrow \tilde{r}_{i j k}=r_{i j k}^{C}+f\left(r_{i j k}^{C}\right) \cdot \xi(i, j, k)$,

where

$\xi(i, j, k)=\frac{2 i(i-N)}{L} \frac{2 j(j-N)}{L} \frac{2 k(k-N)}{L}$.

The function $f$ is given by

$f\left(r_{i j k}^{C}\right)=\frac{\lambda}{1+\exp \left(\mu\left(r_{i j k}^{C}-v\right)\right)}$.

This function resembles the Fermi-Dirac distribution known from statistical mechanics. The parameters $\lambda, \mu$ and $\nu$ are used to control the curvature of the modified grid. Hence, the positions of the modified grid points are given by

$\underline{\tilde{r}}_{i j k}=\tilde{r}_{i j k} \underline{e}=\tilde{r}_{i j k} \frac{\underline{r}_{i j k}^{C}}{r_{i j k}^{C}}$. 

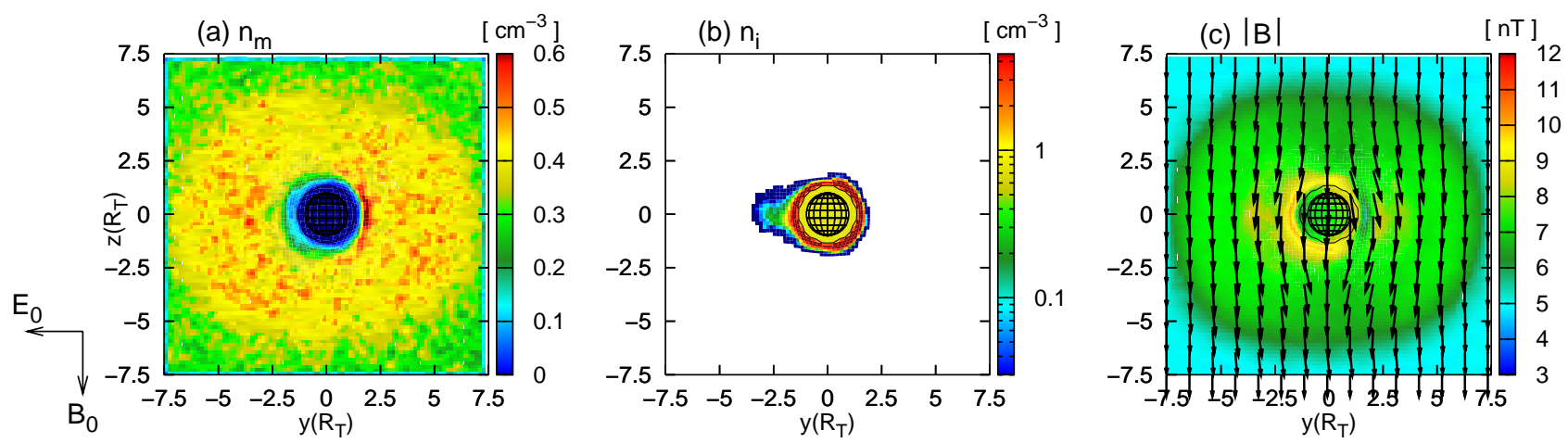

Fig. 6. Interaction of Titan's ionosphere with a supersonic plasma flow - results of a 3-D hybrid simulation. The figure displays the simulation results for a cut through the $(y, z)$ plane of the simulation domain, coinciding with the terminator plane of the obstacle. The undisturbed plasma flow is directed in positive $x$ direction, i.e. out of the paper plane, whereas the undisturbed magnetic field is orientated antiparallel to the $z$ axis. The figure shows (a) the mean $\left(N^{+} / H^{+}\right)$plasma density, (b) the ionospheric plasma density and (c) the magnetic field. The color scale denotes the field strength, whereas the arrows indicate the projection of the magnetic field vectors on the cutting plane. Due to the upstream plasma flow being supersonic, a bow shock arises in front of the obstacle. Since the phase velocity of the fast magnetosonic mode is not isotropic, but depends on the angle between wave propagation and the magnetic field, the shock structure in the terminator plane is not circular. The elliptic structure of the bow shock is clearly identifiable in the magnetic field signature, cf. (c).

As can be seen from Eq. (29), the modified grid is asymptotically Cartesian at the outer boundaries of the simulation box, i.e.

$\xi(i=0, j, k)=\xi(i, j=0, k)=\xi(i, j, k=0)=0$

and

$\xi(i=N, j, k)=\xi(i, j=N, k)=\xi(i, j, k=N)=0$

The resulting curvilinear grid is shown in Fig. 4 for the parameters $\lambda=15.0 R_{T}, \mu=0.7, \nu=0.35$. As can be seen, the grid possesses a spherical symmetrically structure in the vicinity of the obstacle, whereas with increasing distance to the center, it slightly transforms into a Cartesian grid. This type of grid has already been used successfully to study the interaction of the solar wind with the Martian ionosphere (Bößwetter et al., 2004).

\section{Simulation results}

In order to understand the mechanism of Titan's interaction with the subsonic Saturnian magnetospheric plasma flow, it is helpful to discuss the interaction between Titan's ionosphere and a supersonic plasma flow at first. This situation qualitatively resembles the characteristics of the Martian plasma environment which have been discussed in detail by Bößwetter et al. (2004).

\subsection{Titan in supersonic flow}

The simulation geometry for both supersonic upstream flow and the 18:00 Saturnian local time scenario is shown in Fig. 5. The velocity vector of the undisturbed plasma flow is orientated parallel to the $x$ axis, yielding a convective electric field that is directed antiparallel to the $y$ axis. When Titan is located inside the Saturnian magnetosphere, the $y$ axis points towards Saturn.

The results for the quasi-stationary state of the simulation are displayed in figs. 6, 7 and 8 , respectively. The mean plasma density $n_{m}$ of the $\left(N^{+} / H^{+}\right)$plasma ${ }^{1}$ as well as the mean ionospheric ion density $n_{i}$ and the magnetic field for the terminator plane are shown in Fig. 6. In all vector plots, the color scale denotes the field strength, whereas the arrows indicate the projections of the respective vector field on the cutting plane. The results for the polar plane are shown in Fig. 7. Specifically, the color plots display the mean density as well as the mean velocity of both the $\left(N^{+} / H^{+}\right)$plasma and the ionospheric plasma in the vicinity of the obstacle. Besides, the magnetic and electric field configurations are shown. In an analogeous manner, the simulation results for the equatorial plane are shown in Fig. 8.

Due to the impinging plasma flow being supersonic, a bow shock is formed in front of the obstacle. As can be seen from Fig. 6, the bow shock does not possess a spherical, but an elliptical shape in the terminator plane. This asymmetry emerges from the anisotropic propagation velocity of the involved wave mode: The phase speed of the fast magnetosonic mode depends on the angle between the directions of wave propagation and the magnetic field vector. Besides,

\footnotetext{
${ }^{1}$ In all simulation results presented in this paper, the index " $m$ " denotes the magnetospheric $\left(N^{+} / H^{+}\right)$plasma component. Of course, the $\left(\mathrm{N}^{+} / \mathrm{H}^{+}\right)$plasma cannot be interpreted as magnetospheric plasma in the supersonic case. Neverless, the abbreviation " $m$ " has been chosen to keep the notation consistent with all other results concerning this plasma component.
} 

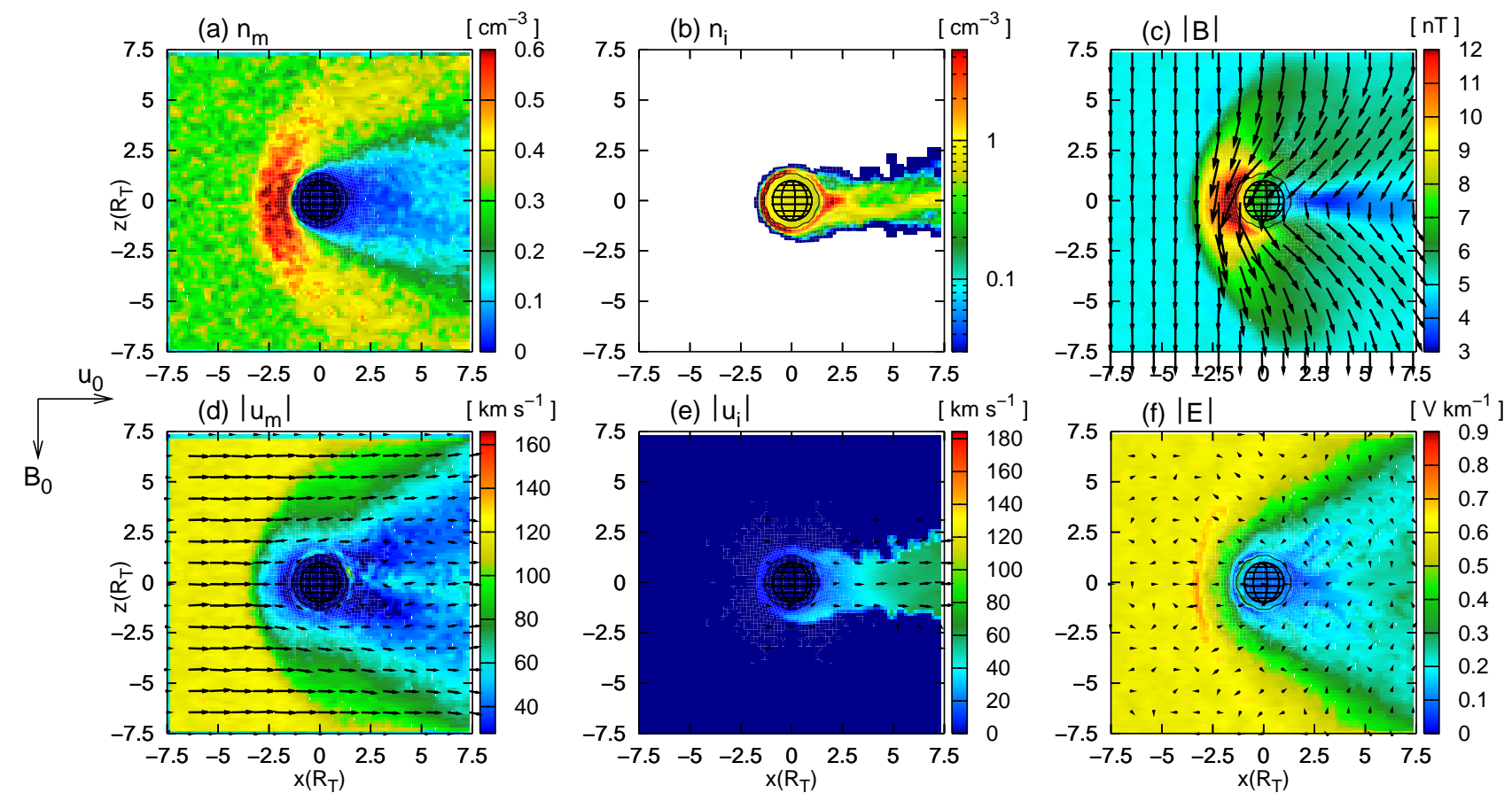

Fig. 7. Interaction of Titan's ionosphere with a supersonic plasma flow - cut through the $(x, z)$ plane of the simulation box which coincides with Titan's polar plane. The undisturbed plasma velocity vector $\underline{u}_{0}$ is parallel to the positive $x$ axis; the background magnetic field $\underline{B}_{0}$ points in $(-z)$ direction. The figure shows (a) the mean $\left(N^{+} / H^{+}\right)$plasma density, (b) the ionospheric plasma density, (c) the magnetic field, (d) the mean $\left(\mathrm{N}^{+} / \mathrm{H}^{+}\right)$velocity, (e) the mean ionospheric ion velocity and (f) the electric field in the cutting plane. The magnetic field clearly drapes around the obstacle. On the one hand, this process results in the formation of an extended magnetic pile-up region at Titan's dayside; on the other hand, two pronounced magnetic lobes are formed in the downstream region. As displayed in the density plots, the $\left(N^{+} / H^{+}\right)$ plasma and the ionospheric ion population scarcely mix in the tail region. The separating boundary is called ion composition boundary. In the tail region, the ionospheric ions become the predominant species. Due to these ions being considerable slower than the ambient plasma flow, an extended region of reduced (convective) electric field strength is formed downstream of the obstacle. In general, the structure of the interaction region is symmetric in the displayed cutting plane, wheras a strong asymmetry with respect to the direction of the convective electric field occurs in the equatorial plane of the obstacle.

the structure of the bow shock is asymmetric with respect to the polar plane. This asymmetry results from a reduction of the downstream fast mode velocity due to the incorporation of the escaping ionospheric ions into the plasma flow (mass loading).

As can be seen from Fig. 7c, the magnetic field exhibits a strong draping pattern. An extended magnetic pile-up region is formed at the dayside of the obstacle, whereas the draping results in the formation of two magnetic lobes in the downstream region. Between these lobes, the magnetic field strength lies significantly below the undisturbed upstream value.

A wake region denoting a significantly reduced $\left(N^{+} / H^{+}\right)$ density is formed downstream of the obstacle. As can be seen from Fig. 7, the $\left(\mathrm{N}^{+} / \mathrm{H}^{+}\right)$plasma and the ionospheric plasma scarcely mix. In the vicinity of Titan, the $\left(N^{+} / H^{+}\right)$density is high in regions where the density of ionospheric ions is low and vice versa. The boundary layer between both plasma species is often called the
Ion Composition Boundary (ICB). A similar separation of both plasma populations is also characteristic for the interaction of the Martian ionosphere with the solar wind (Bößwetter et al., 2004).

As can clearly be seen from Figs. 7 and 8 , the $\left(\mathrm{N}^{+} / \mathrm{H}^{+}\right)$ ion density is significantly reduced in regions where the $\mathrm{N}_{2}^{+}$ ions are the predominant species. However, the structure of the interaction region is highly symmetric in the polar plane, whereas an asymmetry with respect to the direction of the convective electric field $\underline{E}_{0}=-\underline{u}_{0} \times \underline{B}_{0}$ occurs in the equatorial plane. In the following, the half space where $\underline{E}_{0}$ points towards Titan, will be refered to as $E^{-}$hemisphere, whereas the hemisphere where the electric field $\underline{E}_{0}$ is directed away from Titan will be called the $E^{+}$hemisphere. In the inner-magnetospheric simulations discussed in this work, the $E^{-}$hemisphere is always Titan's Saturnfacing hemisphere. In the equatorial plane, a sharp boundary layer between the ionospheric ion tail and the $\left(\mathrm{N}^{+} / \mathrm{H}^{+}\right)$ plasma manifests in the $E^{-}$hemisphere. Besides, the $N_{2}^{+}$ 

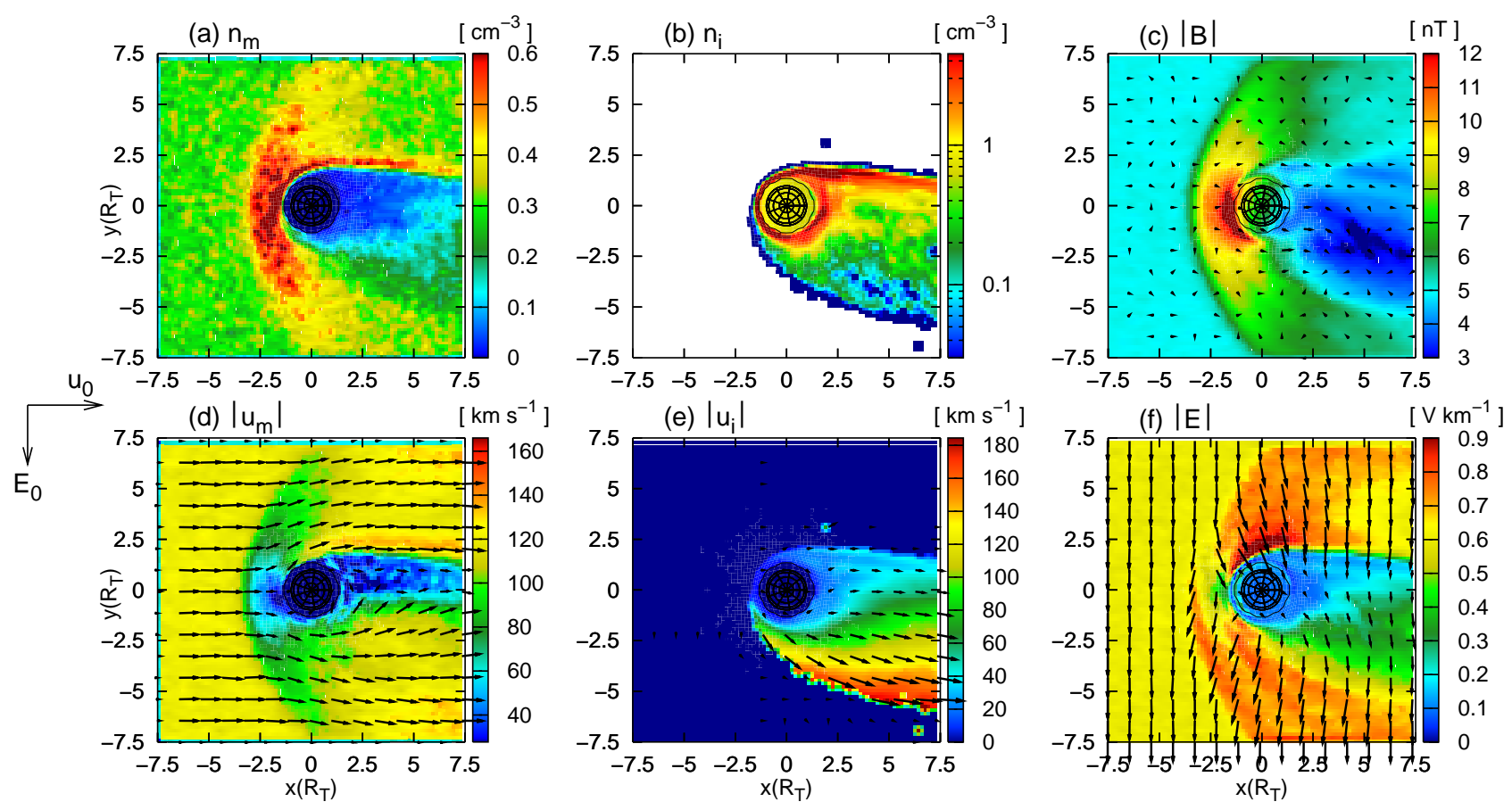

Fig. 8. Interaction of Titan's ionosphere with a supersonic plasma flow - cut through the $(x, y)$ plane of the simulation box which coincides with Titan's equatorial plane. The undisturbed plasma flow is directed in $(+x)$ direction, whereas the background convective electric field $\underline{E}_{0}$ is antiparallel to the $z$ axis. The figure shows (a) the mean $\left(N^{+} / H^{+}\right)$plasma density, (b) the ionospheric plasma density, (c) the magnetic field, (d) the mean $\left(N^{+} / H^{+}\right)$velocity, (e) the mean ionospheric ion velocity and (f) the electric field in the cutting plane. The simulation results exhibit an asymmetry between both hemispheres. The ionospheric tail as well as the region of reduced electric field strength cover a large area in the $E^{+}$hemisphere, whereas they are confined by a sharp boundary layer in the $E^{-}$hemisphere. In the $E^{+}$hemisphere, an extended pick-up region is formed where ionospheric ions are incorporated into the ambient plasma flow. Since their velocity is comparable to the ambient plasma velocity, the pick-up ions make a significant contribution to the convective electric field. For this reason, the decrease of electric field strength in the tail region is less strong than in the $E^{-}$hemisphere. In the $E^{+}$hemisphere, the convective electric field points inward the ionospheric tail preventing these ions from crossing the ICB from inward to outward and mixing with the ambient plasma flow. In this half space, the sharp decrease of ionospheric ion density at the ICB gives rise to a strong electron pressure gradient pointing inward the tail region. Hence, the ambient plasma is forbidden to cross the ICB from outward to inward by the electric field arising from the negative electron pressure gradient. As can be seen from (d), in the $E^{-}$hemisphere the plasma flow is almost tangential to the boundary layer. In contrast to the situation in the $E^{-}$hemisphere, the convective electric field points away from the ionospheric tail in the $E^{+}$hemisphere. Hence, the focussing effect being responsible for the sharp separation of both plasma populations in the $E^{-}$hemisphere does not occur and the boundary layer cannot be formed. The electric force acting on the ionospheric ions makes them mix with the ambient plasma flow where they are picked up and begin to perform a cycloidal motion in $(+x)$ direction. This gives rise to the extended ionospheric tail region in the $E^{+}$hemisphere. Both the convective electric field and the field resulting from the electron pressure gradient are essential for the occurence of asymmetries in the structure of the ICB.

tail does not expand significantly into this hemisphere. As displayed in Fig. 7d, the $\left(N^{+} / H^{+}\right)$plasma in the $E^{-}$hemisphere flows almost tangential to the boundary layer between both populations. On the other hand, in the $E^{+}$hemisphere, the ionospheric tail possesses a diameter of about five Titan radii. Furthermore, the boundary between both plasma populations is less pronounced in this hemisphere; the ionospheric density decreases in a slight way where the $\left(\mathrm{N}^{+} / \mathrm{H}^{+}\right)$ions become predominant and their density similarly increases in a slight manner.

As will be discussed in the following, the convective electric field $\underline{E}=-\underline{u} \times \underline{B}$ is a major condition for this asymmetry to arise. In the tail region, the electric field is significantly weaker than the background field in the undisturbed, homogeneous plasma outside the interaction region. As can be seen in Figs. $7 \mathrm{f}$ and $8 \mathrm{f}$, the region characterized by a reduced electric field strength is symmetric in the polar plane, whereas an asymmetry occurs in the equatorial plane. These structures result primarily from the convective electric field term which can be written as

$$
\underline{E}_{\mathrm{conv}}=-\left\{\frac{n_{m}}{n_{m}+n_{i}} \underline{u}_{m}+\frac{n_{i}}{n_{m}+n_{i}} \underline{u}_{i}\right\} \times \underline{B} .
$$



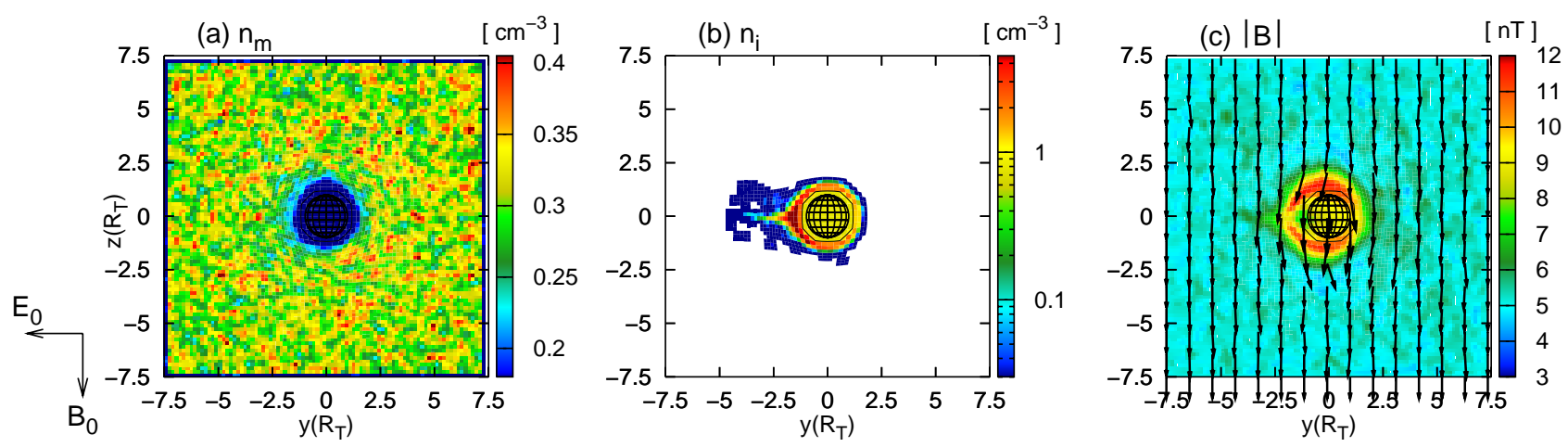

Fig. 9. Interaction of Titan's ionosphere with the subsonic Saturnian magnetospheric plasma at 18:00 local time. The figure displays the results for a cut through the $(y, z)$ plane of the simulation box which coincides with Titan's terminator plane. The undisturbed magnetospheric plasma flow is directed in $(+x)$ direction, i.e. out of the paper plane. The background magnetic field $\underline{B}_{0}$ is orientated antiparallel to the $z$ axis and thus, the convective electric field $\underline{E}_{0}$ points in $(-y)$ direction. The figure displays (a) the magnetospheric ion density $n_{m},(\mathbf{b})$ the ionospheric ion density $n_{i}$ and (c) the magnetic field. Again, the arrows denote the projection of the field vectors in the cutting plane. Since the upstream plasma flow is subsonic, no bow shock is formed in front of the obstacle. As in the case of a supersonic flow, the obstacle's ionospheric tail is more extended in the $E^{+}$hemisphere than in the $E^{-}$hemisphere. Due to Saturn's intrinsic magnetic field being draped around the obstacle, a magnetic pile-up region which is characterized by an increased field strength arises in the immediate vicinity of Titan.
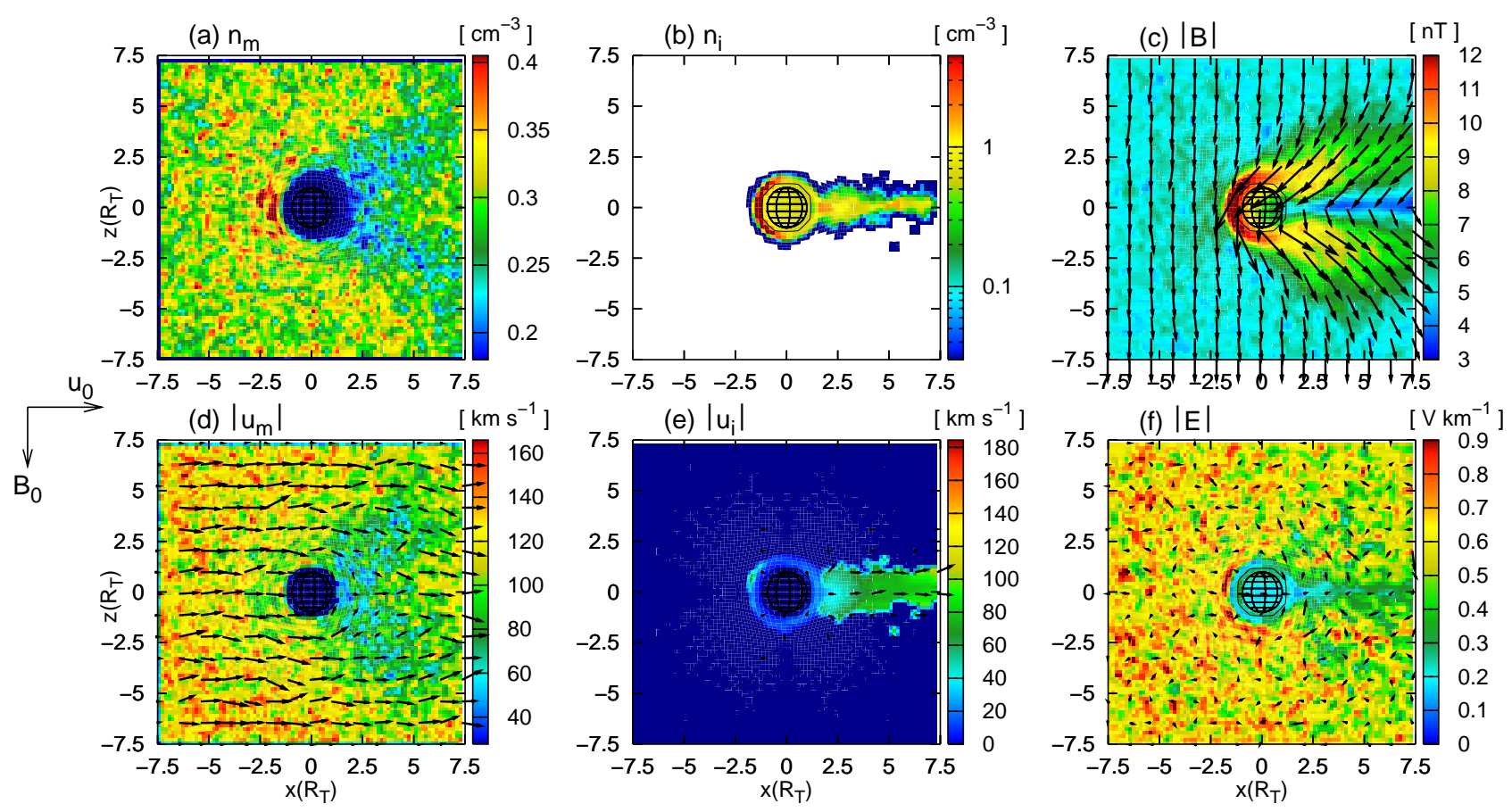

Fig. 10. Interaction of Titan's ionosphere with the subsonic Saturnian magnetospheric plasma at 18:00 local time. The figure shows a cut through the $(x, z)$ plane which coincides with Titan's polar plane. The undisturbed plasma velocity points in $(+x)$ direction; the background magnetic field is antiparallel to the $z$ axis. The figure displays (a) the magnetospheric ion density, (b) the heavy ion density, (c) the magnetic field, (d) the magnetospheric ion velocity, (e) the ionospheric ion velocity and (f) the electric field. In the polar plane, the interaction region exhibits a symmetric structure. As in the case of supersonic plasma flow, the magnetic field exhibits a strong draping pattern, giving rise to a magnetic pile-up region at Titan's dayside and to two magnetic lobes in the wake region. The region between these lobes is characterized by a significant decrease of magnetic field strength. In contrast to the case of supersonic flow, the magnetospheric plasma is no longer separated from the ionospheric tail by an ion composition boundary. Due to both plasma components contributing to the convective electric field, only a slight reduction of electric field strength occurs in the downstream region. 

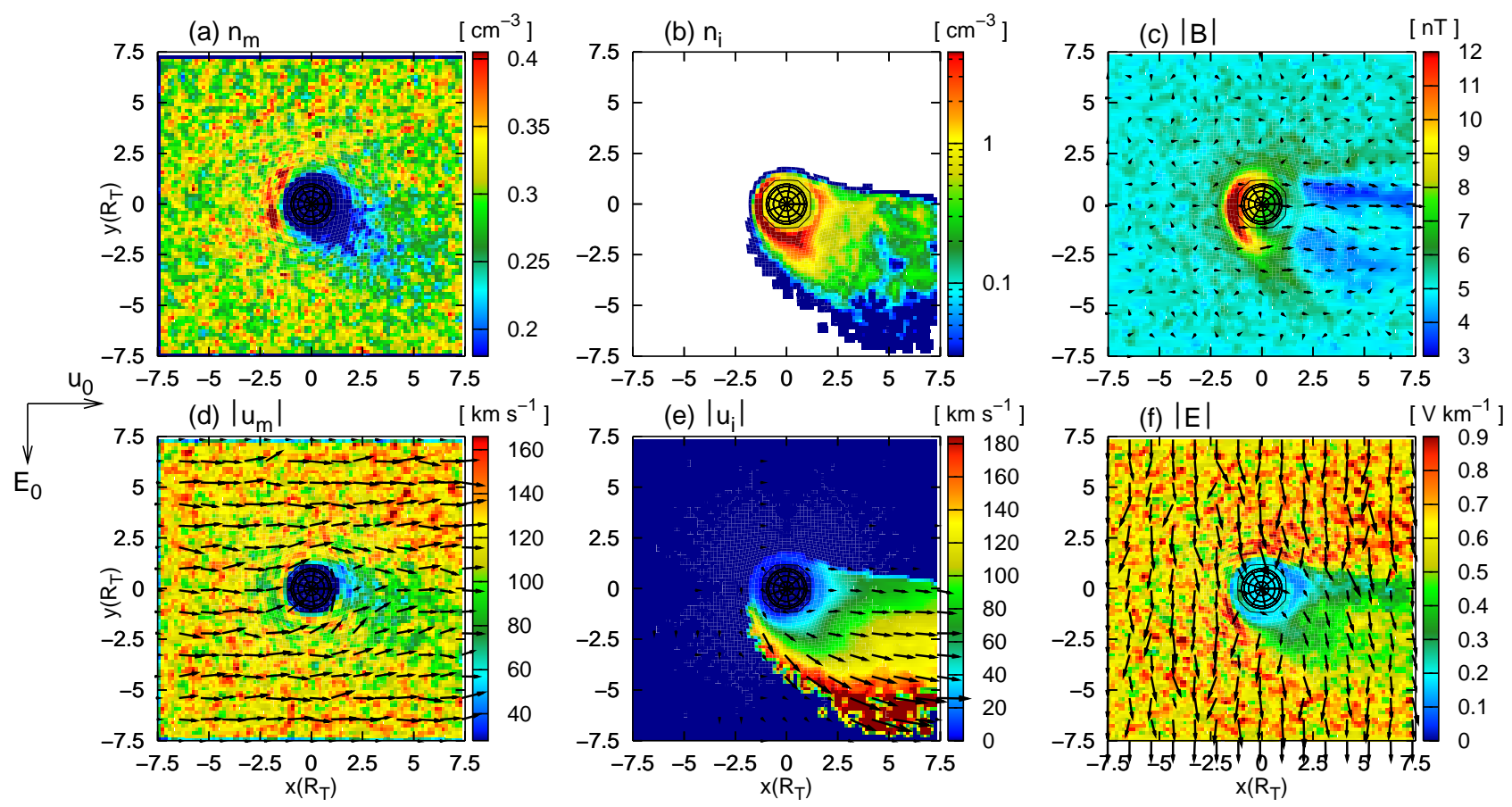

Fig. 11. Interaction between Titan's ionosphere and the subsonic Saturnian magnetospheric plasma flow at 18:00 local time. The figure displays a cut through Titan's equatorial plane. The undisturbed plasma flow is directed in $(+x)$ direction, whereas the convective electric field $\underline{E}_{0}$ is orientated antiparallel to the $z$ axis. In analogy to the case of supersonic flow, the structure of the interaction region exhibits a strong asymmetry between both hemispheres. The convective electric field, being antiparallel to the $y$ axis, forbids the ionospheric tail to expand into the $E^{-}$hemisphere. On the other hand, the electric force drags the ions away from the obstacle in the $E^{+}$hemisphere, resulting in the formation of an extended pick-up region. However, in the $E^{-}$hemisphere, the increase of ionospheric density at the outer flank of the tail is not as sharp as in the case of supersonic flow. Due to the resulting electron pressure gradient being significantly weaker than in the supersonic case, the magnetospheric ions are no longer prevented from entering the tail region and mixing with the ionospheric plasma. For this reason, only a slight, but still asymmetric decrease of magnetospheric plasma density occurs downstream of the obstacle. In regions where the ionospheric ion velocity is low, an area of reduced electric field strength is formed. On the other hand, in the $E^{+}$hemisphere the ionospheric velocity is comparable to the ambient plasma velocity and hence, these ions are able to make a significant contribution to the convective electric field. For this reason, the reduction of electric field strength diminishes in regions where the ionospheric particles are accelerated due to the pick-up process.

In the polar plane, the $\left(\mathrm{N}^{+} / \mathrm{H}^{+}\right)$ions are forbidden to enter the tail region and thus, the convective electric field is mainly governed by the motion of the predominant ionospheric ions. Due to their velocity being considerable smaller than the ambient plasma velocity (cf. Figs. 7d and 8e), a cavity denoting a reduced convective electric field strength is formed in the downstream region. A similar process occurs in the equatorial plane. However, in this plane, an extended pick-up region where ionospheric ions are incorporated into the ambient plasma flow occurs in the $E^{+}$hemisphere. In this area, the pick-up ion velocity becomes comparable to the ambient plasma velocity and hence, the accelerated pick-up ions are able to make a significant contribution to the convective electric field. For this reason, a significant reduction of electric field strength occurs only in the immediate vicinity of the $E^{-}$hemisphere's ion composition boundary where the pick-up ion velocity is negligible. In contrast to this, the slight reduction of $\left(\mathrm{N}^{+} / \mathrm{H}^{+}\right)$density in the $E^{+}$hemisphere is compensated by the presence of fast pick-up ions. In order to understand the occurence of the asymmetric structures in the equatorial plane, the electric field signatures in the vicinity of the ion composition boundary, i.e. near the outer flanks of the ionospheric ion tail, are of major importance. In the $E^{-}$hemisphere, the convective electric field outside the ICB is directed perpendicular to the boundary, pointing inside the tail. Hence, the ionospheric ions are forbidden to cross the ICB from inward to outward. This results in the formation of a sharp boundary layer denoting a significant density jump at the flank of the tail. On the other hand, the resulting electron pressure gradient is directed inward the ICB. Hence, the ambient plasma is prevented from crossing the ICB from outward to inward due to the electric field arising from the $-\nabla P_{e, i}$ term. 


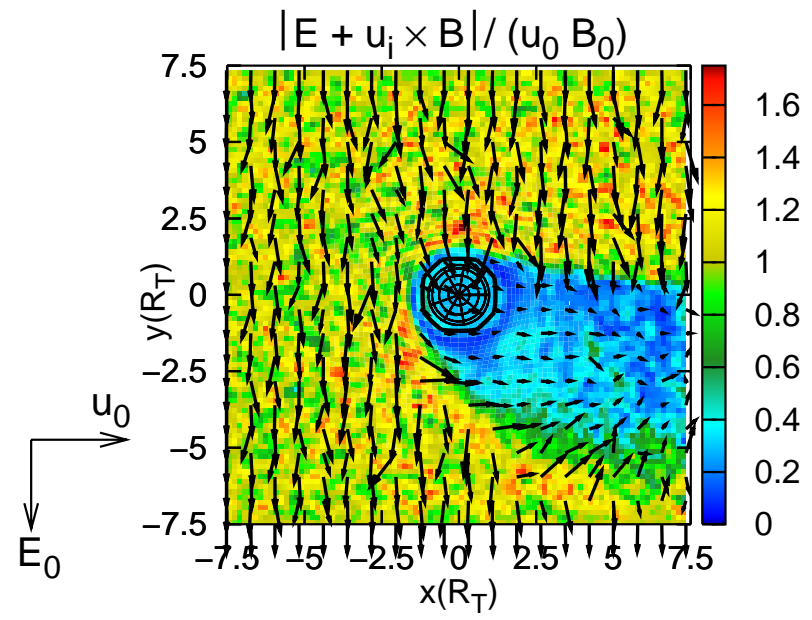

Fig. 12. Lorentz force acting on ionospheric ions (18:00 Saturnian local time). For a cut through the terminator plane, the figure displays $\underline{E}+\underline{u}_{i} \times \underline{B}$ in normalized units. In regions where the ionospheric density does not vanish, this quantity is the average Lorentz force acting on the ionospheric ions. In regions with $n_{i} \approx 0$, the quantity displayed in the figure is identical to the electric field. Since the Lorentz force is orientated perpendicular to the upper flank of the ionospheric tail and points inward the tail, it is responsible for preventing the tail from expanding into the $E^{-}$hemisphere. The figure also illustrates the acceleration of the ionospheric nitrogen ions parallel to the direction of $\underline{E} \times \underline{B} \uparrow \uparrow \underline{u}_{0}$ at the tail's outer flank in the $E^{+}$hemisphere.

A quite different situation occurs in the $E^{+}$hemisphere. Again, the convective electric field is directed perpendicular to the flank of the ionospheric tail, but in this half space, it points outward the tail region. Thus, the $N_{2}^{+}$ions are dragged away from the ionosphere and are picked up by the ambient plasma flow. They can mix with the surrounding flow and form a cycloidal tail. This is the major reason for the ICB not to be sharply developed in the $E^{+}$hemisphere. To sum up the major result, the convective electric field as well as the $-\nabla P_{e}$ term have to be considered essential for the ICB to be formed.

Finally, the results shall be compared with another hybrid simulation study carried out by Kallio et al. (2004). The authors have used a 3-D hybrid code to examine the interaction of Titan's ionosphere with a super-Alfvénic, supersonic and supermagnetosonic plasma flow. However, this model neglects the electron pressure term in the generalized Ohm's law and consequentely, the electron temperature is set to zero. Nevertheless, the results of this study are at least in rough agreement with the simulations presented above. The formation of a bow shock as well as a magnetic pileup region in front of the obstacle and an induced magnetotail on the wakeside are reproduced by both simulation codes. Moreover, Kallio et al. (2004) also show that the density of the ambient plasma is low in the center of the tail where the

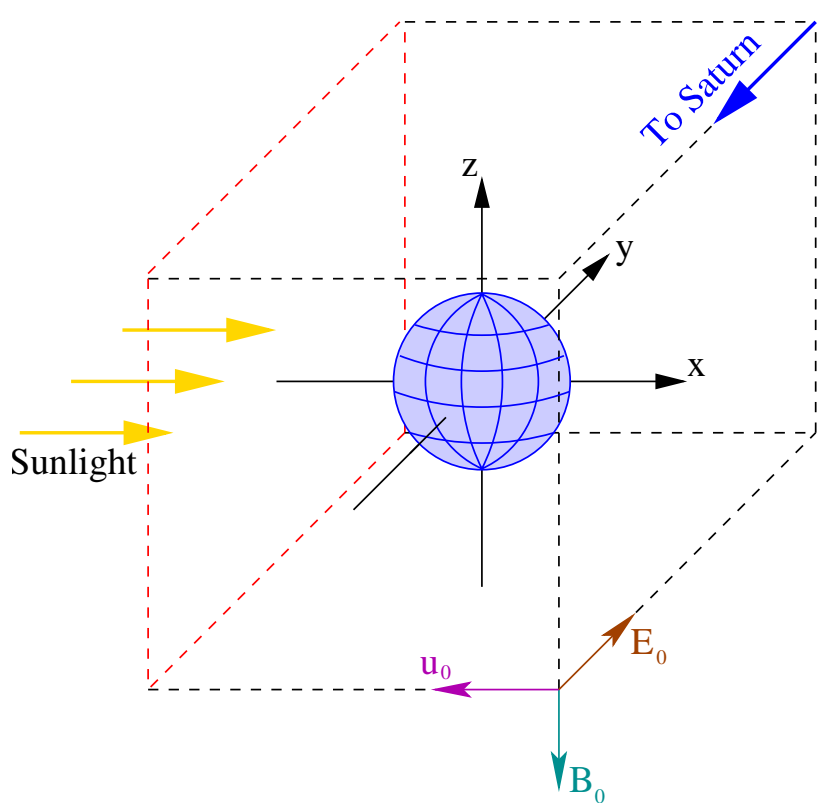

Fig. 13. Titan's plasma environment at 06:00 Saturnian local time simulation geometry. The center of Titan coincides with the center of the cubic simulation domain. Again, the positive $x$ axis is parallel to the direction of the solar UV radiation, whereas the undisturbed Saturnian magnetic field is orientated antiparallel to the $z$ axis. Hence, the velocity vector of the undisturbed corotating plasma flow is directed antiparallel to the $x$ axis. The convective electric field points in $(+y)$ direction, i.e. away from Saturn. The $(x, y)$ plane of the coordinate system coincides with Titan's equatorial plane; the terminator line is located in the $(y, z)$ plane.

ionospheric ions become predominant. Besides, the simulation model developed by Kallio et al. (2004) also demonstrates the existence of asymmetries in the density of the escaping ions.

\subsection{Titan at 18:00 local time}

The simulation results for a subsonic upstream plasma flow are displayed in Figs. 9, 10 and 11, respectively. The magnetic field strength as well as the mean magnetospheric and ionospheric plasma densities in the terminator plane are displayed in Fig. 9. The characteristic plasma and field parameters for the polar plane are displayed in Fig. 10, whereas Fig. 11 shows the simulation's quasi-stationary state for Titan's equatorial plane. In accordance with the results for the case of a supersonic upstream flow, the structure of the interaction region is symmetric in the polar plane. In both the subsonic and the supersonic case, asymmetries between $E^{-}$ and $E^{+}$hemisphere occur in Titan's equatorial plane.

Due to the upstream plasma flow being subsonic, no bow shock is formed in front of the obstacle. However, Saturn's intrinsic magnetic field clearly drapes around the obstacle, resulting in the formation of two pronounced magnetic lobes 

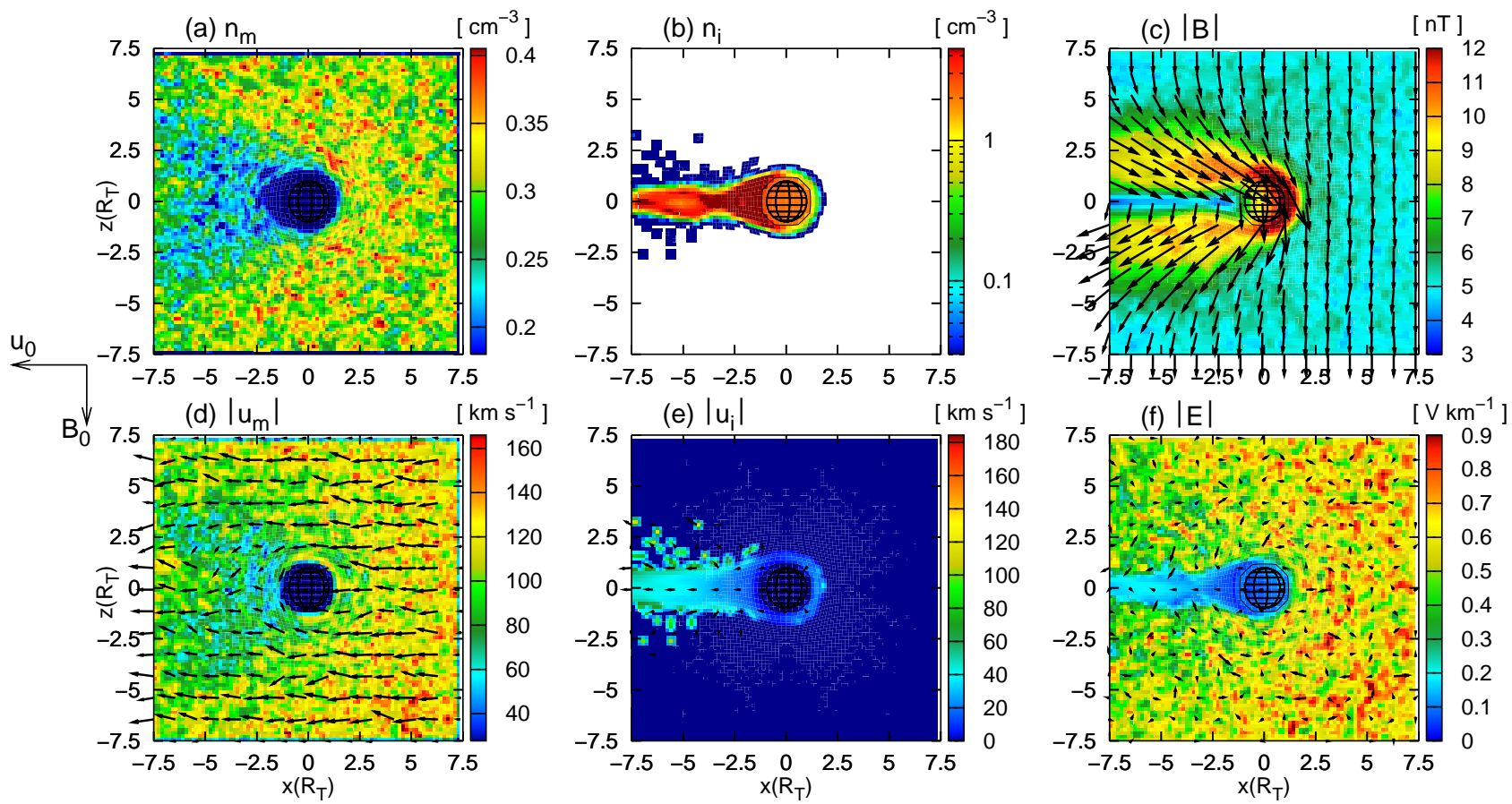

Fig. 14. Titan's plasma environment at 06:00 Saturnian local time - cut through the $(y, z)$ plane of the coordinate System, coinciding with Titan's terminator plane. The figure displays (a) the magnetospheric ion density, (b) the ionospheric nitrogen density, and (c) the magnetic field. The global features of the interaction region, especially the asymmetry of the nitrogen tail with respect to the direction of the convective electric field, are in complete correspondence to the situation at 18:00 local time.

in the wake region and a magnetic pile-up region at Titan's dayside, denoting an increased field strength. Between the two lobes, a region where the magnetic field strength lies significantly below the upstream value of the undisturbed magnetospheric plasma is formed. The magnetotail possesses a spatial extension of about $4 R_{T}$ in $\pm z$ direction and still affects the magnetic field structure at a distance of (at least) $7.5 R_{T}$ from the center of the obstacle. Thus, even in the case of subsonic plasma flow, the presence of Titan has a noticable influence on the magnetospheric plasma flow.

In contrast to the case of supersonic plasma flow, only a slight decrease of plasma density and velocity occurs in the downstream region. Besides, the interaction region is no longer characterized by a clear separation of the magnetospheric and ionospheric plasma component. Furthermore, the interaction region does not exhibit a pronounced outer boundary.

Only a slight reduction of the electric field strength manifests in the tail region. On the one hand, the decrease of $E$ is not as significant as in the case of supersonic flow because the magnetospheric ion density downstream of the obstacle does no longer vanish and hence, the magnetospheric ions can make a significant contribution to the convective electric field. On the other hand, an extended pick-up region arises in the $E^{+}$hemisphere of the equatorial plane. In this area, the ionospheric ions are accelerated in the direction of
$\underline{E} \times \underline{B}$, their velocity being comparable to that of the magnetospheric plasma. Because of the resulting contribution to the convective electric field, only a minor decrease of $E$ occurs in the $E^{+}$hemisphere. By comparing the structure of the ionospheric tail in Fig. 11e with the shape of the area denoting a reduced electric field strength in Fig. 11f, it can clearly be seen that the reduction of electric field strength diminishes in exactly the same regions where the $N_{2}^{+}$velocity increases due to the pick-up process. In contrast to this, only a minor acceleration of the ionospheric ions occurs in the $E^{-}$hemisphere. In this half space, the slight reduction of electric field strength arises from the decrease of the magnetospheric ion density and velocity in the downstream region. The decrease of electric field strength manifesting in the polar plane has to be explained in an analogeous manner since the heavy ion velocity in the downstream region is sigificantly smaller than in the equatorial plane. In the polar plane, the $N_{2}^{+}$ions form a narrow tail whose diameter is of the order of only two Titan radii, as to be seen in Figs. 10b and e.

The mechanism giving rise to the asymmetric structure of the interaction region in the equatorial plane can be explained in analogy to the previous discussion of the supersonic case. Again, the convective electric field at the outer flanks of the ionospheric tail has to be considered to be of major consequence. On the on hand, in the $E^{-}$hemisphere, this field is directed perpendicular to the ionospheric tail pointing inward 

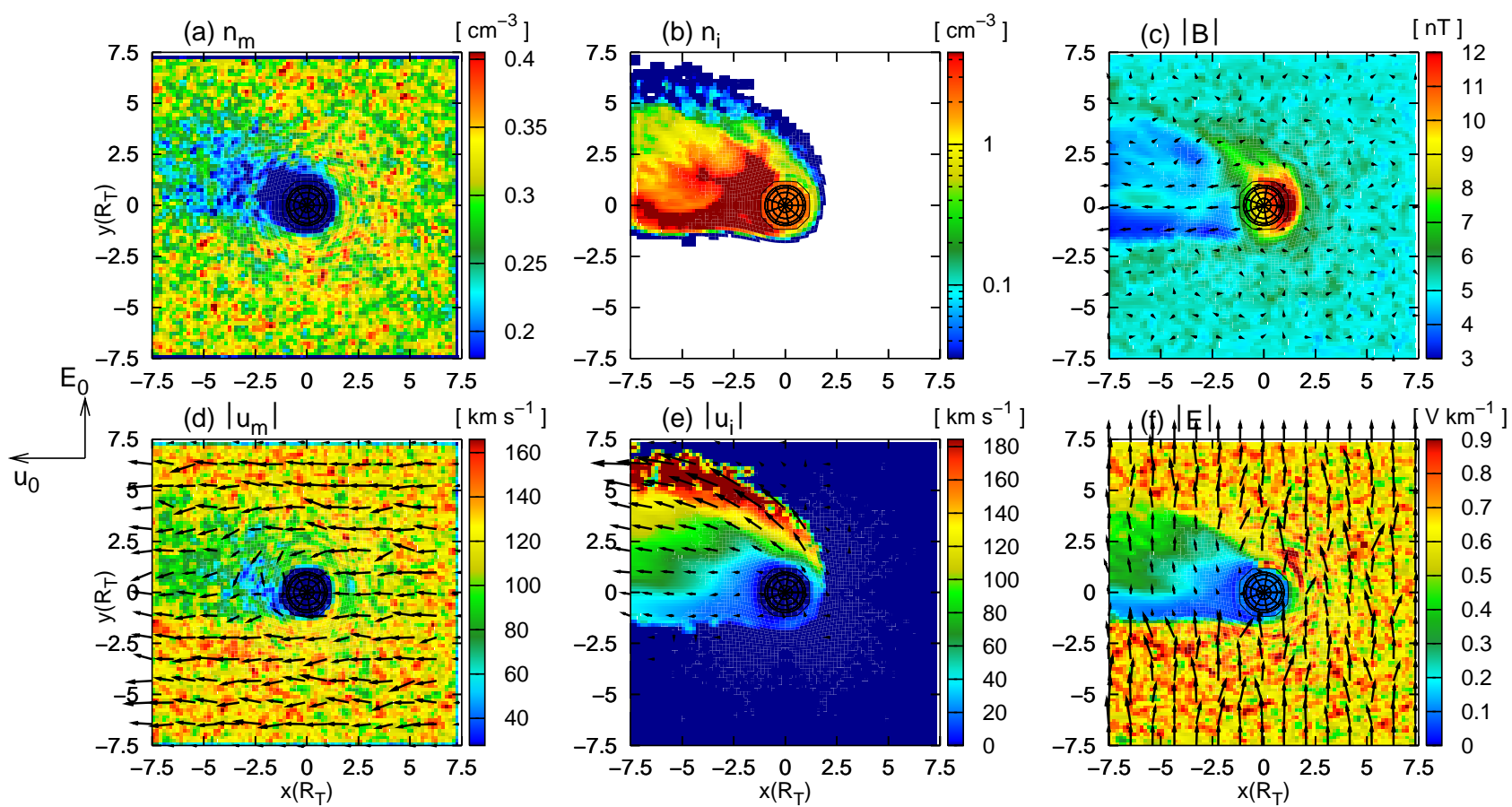

Fig. 15. Titan's plasma environment at 06:00 Saturnian local time. The figure displays the magnetospheric and ionospheric ion densities in (a) and (b), and velocities in (c) and (e), as well as the electromagnetic field quantities in (c) and (f) for a cut through the $(x, z)$ plane of the coordinate system. The simulation results show the formation of a strong magnetic draping pattern and a slight reduction of both magnetospheric ion density and velocity in the downstream region. Due to the dayside of the obstacle being located in the wake region, the ionospheric nitrogen density in the tail is about four times higher than in the 18:00 LT scenario. Again, the interaction region exhibits an asymmetric structure in the plane parallel to the convective electric field.

it. In exactly the same manner as in the case of a supersonic upstream flow, the focussing effect arising from the electric force on the ions forbids the heavy ion tail to expand in the $E^{-}$hemisphere. On the other hand, the convective electric field in the $E^{+}$hemisphere points away from the ionosphere and thus, it drags the ionospheric ions away from the obstacle. These ions are incorporated into the magnetospheric plasma flow, resulting in the formation of an extended pickup region in the $E^{+}$hemisphere.

The crucial question is why both plasma components are no longer clearly separated from each other. As discussed in the previous section, the electric force emerging from the negative electron pressure gradient is most important for the formation of a sharply pronounced ion composition boundary in the $E^{-}$hemisphere. Due to a sharp decrease of ionospheric ion density occuring near the outer flank of the ionospheric tail in the $E^{-}$hemisphere, the resulting force is capable of preventing the ambient plasma from entering the ionospheric tail in the case of supersonic flow. However, the ionospheric density in the $E^{-}$hemisphere decreases only in a slight manner when the upstream flow is subsonic. Hence, the resulting electron pressure gradient at the outer flank of the tail is significantly weaker than in the supersonic case and can no longer forbid the magnetospheric plasma to cross the outer boundary of the ionospheric tail from outward to inward. Nevertheless, as can be seen from Fig. 11f, at least a weak decrease of magnetospheric plasma density occurs in the equatorial plane, being less pronounced in the $E^{-}$ hemisphere than in the $E^{+}$hemisphere. This structure indicates that the electron pressure gradient at the outer flank of the ionospheric tail in the $E^{-}$hemisphere still influences the magnetospheric ions, preventing at least some of them from entering the ionospheric tail region. The mechanism is also illustrated in Fig. 12, displaying the Lorentz force that acts on nitrogen particles of ionospheric origin. At the ionospheric tail's outer flank in the $E^{-}$hemisphere, this force is directed inward and thus, forbids the tail to expand into the $E^{-}$hemisphere. On the other hand, in the outer regions of the tail in the $E^{+}$hemisphere, the Lorentz force possesses a significant component parallel to the $(+x)$ axis, illustrating the pick-up force acting in the direction of $\underline{E} \times \underline{B}$. Inside the tail region itself, where ions of ionospheric origin become the predominant species, the Lorentz force almost vanishes due to both the mean ion velocity and the electric field being significantly weaker than the undisturbed upstream values.

In general, the basic principle of the mechanism giving rise to the asymmetries in the equatorial plane seems to be the same for the case of a subsonic as well as for a supersonic 


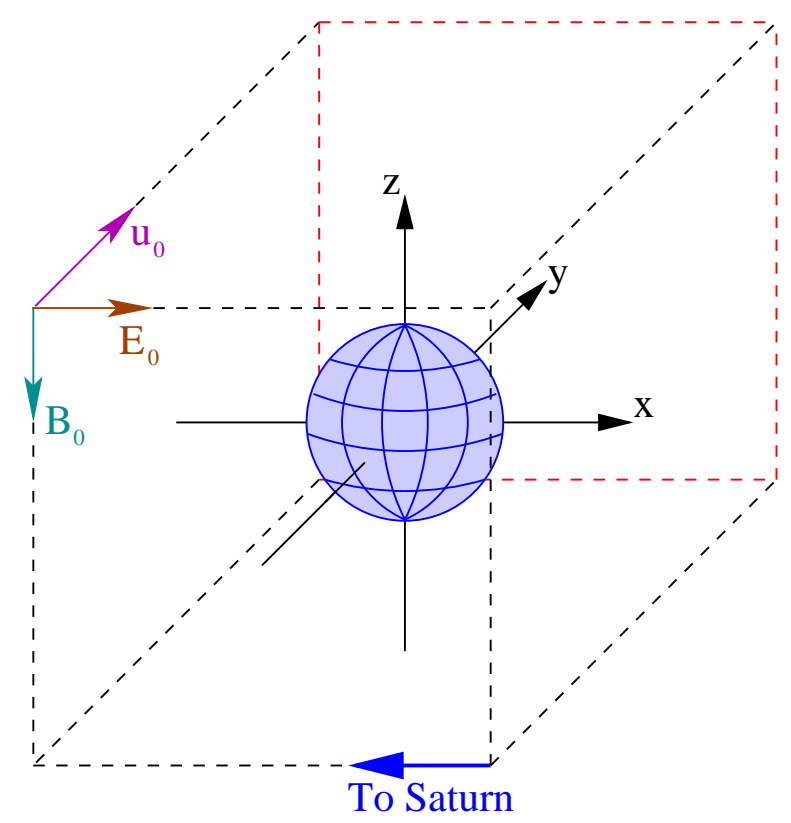

Fig. 16. Titan's plasma environment at 00:00 Saturnian local time simulation geometry. Again, the $x$ axis is pointing from the sun to Titan, whereas the undisturbed magnetic field is orientated antiparallel to the $z$ axis. The undisturbed plasma flow is directed in $(+y)$ direction and yields a convective electric field that is parallel to the $x$ axis, pointing away from Saturn.

upstream plasma flow. In the $E^{+}$hemisphere, the force arising from the convective electric field drags the ionospheric tail away from the obstacle, whereas in the $E^{-}$hemisphere it confines the tail and forbids it to expand. In the supersonic case, this results in the formation of a sharp density jump and hence, a strong electron pressure gradient near the outer flank of the ionospheric tail in the $E^{-}$hemisphere, preventing the surrounding plasma from entering the tail region. On the other hand, the outer boundary of the heavy ion tail is less pronounced and less sharp in the case of subsonic flow, so that the resulting weak pressure gradient is only capable of causing a slight, but still asymmetric decrease of magnetospheric ion density downstream of the obstacle. Although the mechanism generating the asymmetries is the same in both cases, the resulting boundaries are extremely sharp in the supersonic case, whereas no significant separation of magnetospheric and ionospheric plasma -consequently no ion composition boundary- is formed in the case of subsonic plasma flow. Due to the plasma flow being subsonic beyond a bow shock, a qualitative resemblence of the interaction mechanism for the case of subsonic and supersonic upstream flow is absolutely expectable.

The hybrid simulations carried out by Brecht et al. (2000) also show an asymmetric structure of the interaction region. On the one hand, this study showed that the global structure of the interaction region resembles the results obtained from MHD simulations (cf. Cravens et al., 1998; Ledvina and Cravens, 1998; Nagy et al., 2001). On the other hand, the magnetic pile-up region has proven to be more prominent in the direction of the convective electric field. In agreement with the results presented above, Brecht et al. (2000) demonstrate that the ionospheric tail region is not aligned with the undisturbed plasma flow, but is offset towards the $E^{+}$hemisphere of Titan. Recent hybrid simulation results from Kallio et al. (2004) also indicate that the tail structure is not symmetric because the system exhibits a definite preference of forming an extended ion tail in the $E^{+}$hemisphere.

The simulation results are also in qualitative agreement with Cassini measurements of cold plasma in the ionosphere of Titan, as discussed by Wahlund et al. (2005). During the Voyager 1 encounter (cf. Gurnett et al., 1982) as well as during Cassini's passage through Titan's wake on 13 December 2004, a large asymmetry in the structure of the mass-loading region has been detected. In the $E^{+}$hemisphere, plasma of ionospheric origin (indicated by the enhanced number density and slowed ion ram speed) has been detected as far as $9 R_{T}$ away from Titan. Hence, data obtained during this flyby confirm the existence of an extended mass-loading region in the $E^{+}$hemisphere, as it has been proposed by the theoretical models (see also Brecht et al., 2000; Kallio et al., 2004). On the other hand, the mass-loading region's outer boundary in the $E^{-}$hemisphere has shown to be located much closer to Titan than in the anti-Saturn-facing $E^{+}$hemisphere. Nevertheless, the data collected during the first Cassini flyby on 26 October 2004 revealed no large asymmetry in the structure of the mass-loading region (Wahlund et al., 2005). Based on our simulation results, the lack of an asymmetry cannot be explained. However, the existence of an asymmetry is not only confirmed by our study, but its occurence has also been demonstrated by Brecht et al. (2000), by Kopp and Ip (2001) and by Kallio et al. (2004).

\subsection{Titan at 06:00 local time}

The simulation geometry is shown in Fig. 13. The orientations of the $x$ and $z$ axes have been chosen in analogy to the 18:00 LT situation: The $x$ axis is parallel to the direction of the solar UV radiation, whereas the $z$ axis is antiparallel to the homogeneous backgound magnetic field. The simulation results are shown in Figs. 14 and 15. In general, the global features of the interaction region are the same as in the 18:00 LT situation: The magnetic field drapes around the obstacle, giving rise to a magnetic pile-up region at the ramside and two magnetic lobes in the wake region. The results of Brecht et al. (2000) who have performed 3-D hybrid simulations of Titan's interaction with a cold $(T=0 \mathrm{~K})$ magnetospheric plasma also indicate that the global magnetic field topology does not change significantly during Titan's orbital period. Besides, the structure of the interaction region exhibits a strong asymmetry with respect to the direction of the convective electric field since the Lorentz force prevents the 

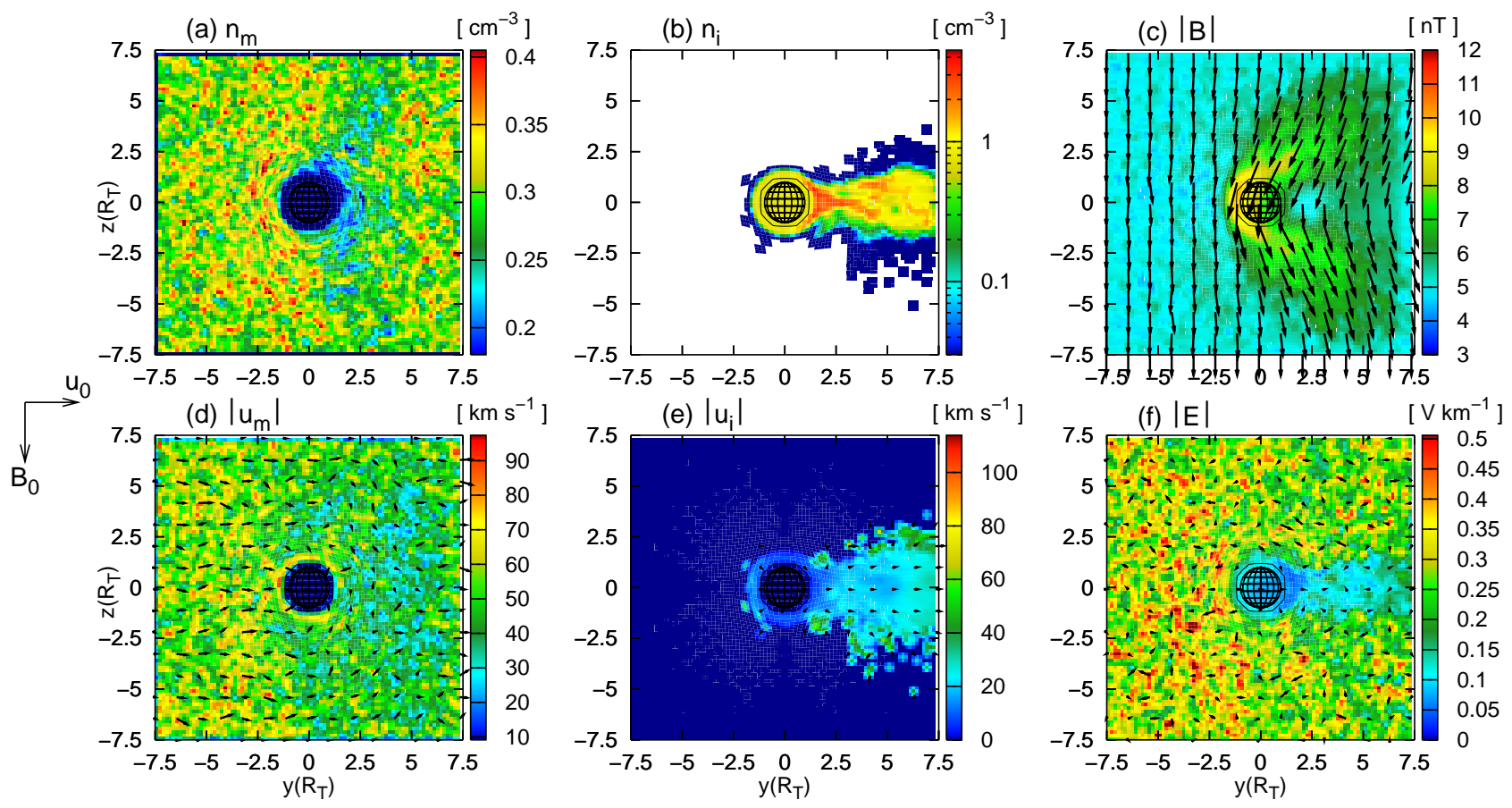

Fig. 17. Titan's plasma environment at 00:00 Saturnian local time. The figure displays a cut through the obstacle's polar plane which coincides with the $(y, z)$ plane of the coordinate system. The figure displays (a) the magnetospheric ion density, (b) the ionospheric nitrogen density, (c) the magnetic field strength, (c) the magnetospheric ion velocity, (e) the ionospheric ion velocity and (f) the electric field. The magnetic draping pattern is not as pronounced as in the 06:00 LT and the 18:00 LT scenario. On the one hand, the magnetic lobes are less confined to the immediate vicinity of Titan; on the other hand, the peak magnetic field strengths in the lobe regions as well as in the pile-up region at Titan's ramside are significantly weaker than in the simulations assuming the upstream plasma flow to be super-Alfvénic. Moreover, the area of reduced magnetic field strength between the two lobes, being characteristic for both the 06:00 LT and the 18:00 LT case, has almost vanished. Compared to the other simulations presented in this work, the characteristic time scale for the formation of the magnetic field pattern is significantly smaller, allowing the field lines to pass the obstacle in the direction perpendicular to the cutting plane. The ionospheric tail's extension perpendicular to the flow direction is larger than in the 06:00 LT and the 18:00 LT simulation. In the downstream region, the electric field strength and the magnetospheric plasma parameters exhibit only a minor reduction.

nitrogen tail from expanding into the $E^{-}$hemisphere. However, due to Titan's dayside being located in the wake region, the nitrogen density in the tail is about a factor 4 higher than in the 18:00 LT case.

However, the results presented by Brecht et al. (2000) suggest that the location of maximum ionospheric production has a significant influence on the global structure of the interaction region. As our simulations do not confirm this significance, it must be assumed that neglecting the ambient plasma temperature has a noticable influence on the structure of the interaction region.

\subsection{Titan at 00:00 local time}

This scenario refers to the case of Titan being located in Saturn's wake at 00:00 Saturnian local time. The simulation geometry is displayed in Fig. 16. Although the satellite is protected from the solar ultraviolet radiation by Saturn, the $x$ axis is still pointing from the sun to Titan in order to be consistent with the other simulations discussed in this work.
In consequence, the undisturbed upstream plasma flow is directed in $(+y)$ direction, yielding a convective electric field that is parallel to the positive $x$ axis. The plasma and field parameters for the polar plane are shown in Fig. 17, whereas Fig. 18 displays the results for Titan's orbital plane which is perpendicular to the undisturbed Saturnian magnetic field.

As to be seen in Fig. 17c, the magnetic draping pattern in the vicinity of Titan is significantly less pronounced than in the simulation results for 06:00 and 18:00 local time, where the upstream plasma flow is assumed to be super-Alfvénic, subsonic and submagnetosonic. In the 00:00 local time scenario, the two magnetic lobes possess an extension of about $\pm 6 R_{T}$ in the direction of the $z$ axis. In contrast to this, the magnetic draping pattern is significantly sharper in the cases of Titan being exposed to a super-Alfvénic plasma flow, its extension in the direction perpendicular to he flow being of the order of $\pm 4 R_{T}$. Moreover, the simulation results for the 06:00 LT as well as for the 18:00 LT scenario show the formation of a pronounced region between the two lobes where 

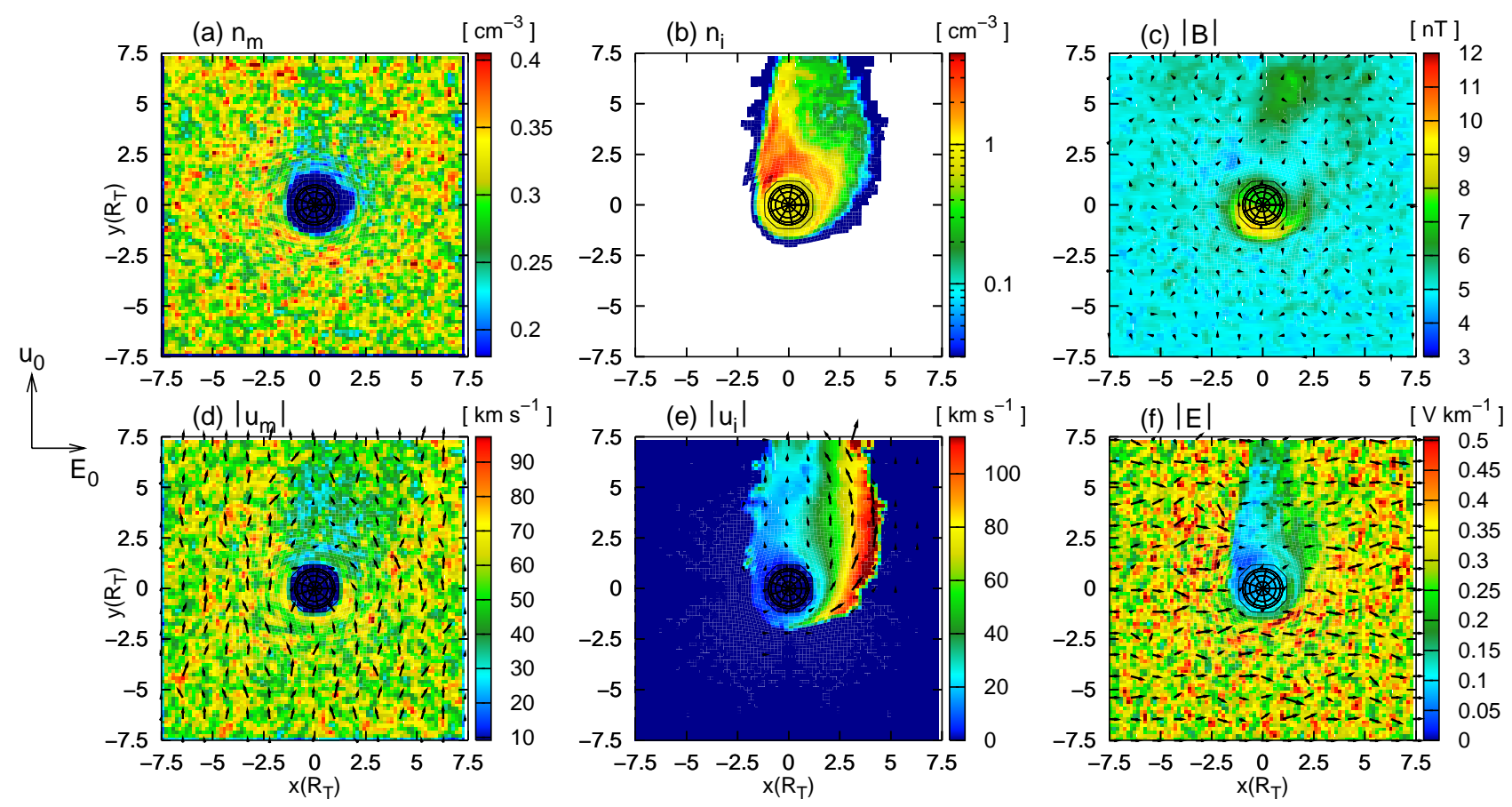

Fig. 18. Titan's plasma environment at 00:00 Saturnian local time. The figure displays a cut through the equatorial plane of Titan which is perpendicular to the undisturbed magnetic field. The figure displays (a) the magnetospheric ion density, (b) the ionospheric nitrogen density, (c) the magnetic field strength, (d) the magnetospheric ion velocity, (e) the ionospheric ion velocity and (f) the electric field. As in the 06:00 LT and the 18:00 LT scenarios, the ionospheric tail exhibits a strong preference to expand into the $E^{+}$hemisphere. However, since the value of the upstream plasma velocity is about $60 \%$ smaller than in the other simulation runs, the convective electric field and hence the Lorentz force that drags the ionospheric ions into the $E^{+}$hemisphere are significantly weaker, yielding a tail diameter of only $5 R_{T}$ in $E^{+}$ direction. In contrast to this, when the upstream flow is super-Alfvénic, particles of ionospheric origin can be found in a distance of more than $7 R_{T}$ perpendicular to the flow direction. Because the ionospheric ion velocity in the tail is comparable to the ambient plasma velocity, only an insignificant decrease of (convective) electric field strength occurs downstream of the obstacle.

the magnetic field strength almost vanishes. As displayed in Figs. $17 \mathrm{c}$ and $18 \mathrm{c}$, the interaction does not give rise to such a structure when Titan is located in Saturn's wake. Besides, in the 00:00 LT scenario, only a minor increase of magnetic field strength occurs in front of the obstacle: Figs. $17 \mathrm{c}$ and $18 \mathrm{c}$ indicate that a peak field strength of about $9 \mathrm{nT}$ is achieved at Titan's ramside, i.e. the magnetic field in this region is around a factor 2 stronger than in the undisturbed upstream plasma flow. In contrast to this, when Titan is located in Saturn's magnetosphere at 06:00 LT or at 18:00 LT, the magnetic pile-up yields a field strength of more than $12 \mathrm{nT}$ at the side of Titan facing the corotating plasma flow. In general, due to the upstream plasma velocity in the 00:00 LT scenario being about $1-0.77 / 1.87=60 \%$ smaller than in the other situations under consideration, the characteristic time scale for the formation of the magnetic lobes in the 00:00 LT simulation is significantly larger than in the case of Titan being exposed to a super-Alfvénic upstream flow. Thus, instead of draping around the satellite, the magnetic field lines are capable of passing the obstacle in the third spatial direc- tion, i.e. they get beyond Titan by evading the obstacle in $( \pm x)$ direction. This aspect also emphasizes that a 2-D simulation code would be incapable of covering all features of Titan's plasma environment.

The only other simulation study refering to the 00:00 LT scenario has been conducted by Ledvina et al. (2004a). As the Mach numbers of the upstream plasma flow have been chosen in complete agreement with this magnetohydrodynamic study, a direct comparison between the results is possible. In complete qualitative agreement with the results discussed in this section, Ledvina et al. (2004a) demonstrate that for the case of Titan being located in Saturn's wake, the magnetic draping pattern is significantly less confined to the immediate vicinity of Titan than in the 18:00 LT scenario. Nevertheless, a quantitative agreement to the results of the magnetohydrodynamic model cannot be achieved. On the one hand, the results presented by Ledvina et al. (2004a) indicate no large difference between the structure of the magnetic pile-up region in the 00:00 LT and the 18:00 LT scenario: in both simulations, the peak magnetic field strength at Titan's 
ramside is about a factor 3-4 higher than the undisturbed upstream magnetic field. Besides, the authors suggest that the peak field strength in the magnetic lobes is also almost the same in both situations. As stated above, these quantitative aspects are not confirmed by the hybrid simulations discussed in this chapter, even though the results concerning the global features of the interaction region are in good qualitative agreement.

As to be seen in Fig. 17b, which displays the ionospheric ion density in the polar plane, the ionospheric tail possesses a larger extension perpendicular to the obstacle than in the 06:00 LT and in the 18:00 LT situation. However, the tail is still symmetric in the polar plane, whereas it exhibits a strong asymmetry with respect to the direction of the convective electric field, as displayed in Fig. 18b. Nevertheless, when Titan is located in Saturn's wake, the tail possesses an extension of only $5 R_{T}$ in the $E^{+}$hemisphere, whereas nitrogen particles of ionospheric origin can be found at a distance of more than $7 R_{T}$ in $E^{+}$direction in both the 06:00 LT and the 18:00 LT scenario. As discussed in the previous chapter, the convective electric field is of major importance for dragging the ionospheric particles away from Titan, leading to the formation of an extended tail region in the $E^{+}$hemisphere. However, since the upstream plasma velocity in the 00:00 LT scenario is about $60 \%$ smaller than in the 06:00 LT and the 18:00 LT run, the convective electric field in the 00:00 LT simulation is significantly weaker than in the other cases under consideration. Thus, the force acting on the ionospheric particles parallel to the $E^{+}$direction is weaker than in the simulations where the upstream flow is super-Alfvénic, leading to the formation of a pick-up region which is less extended in $E^{+}$direction than in the 06:00 LT and the 18:00 LT scenario.

When Titan is located in Saturn's wake at 00:00 LT, only a small cavity denoting a reduced electric field strength arises downstream of the obstacle, as to be seen in Fig. 18f. Besides, the decrease of electric field strength is less significant than in the 06:00 LT and in the 18:00 LT scenario. On the one hand, the slight reduction of magnetospheric plasma velocity in the downstream region causes only a minor decrease of convective electric field strength. On the other hand, this reduction is almost entirely compensated by the ionospheric pick-up ions, whose velocity in the $E^{+}$hemisphere is comparable to or even significantly higher than the undisturbed upstream plasma velocity, giving a significant contribution to the convective electric field. This situation is clearly illustrated in Figs. 18d and 18e, respectively. As displayed in Fig. 18a, in the 00:00 LT scenario, a reduction of magnetospheric plasma density downstream of the obstacle is practically not existent, implying that the electron pressure gradient is too weak to have a noticable influence on the global structure of the interaction region.

\section{Summary}

Titan's plasma environment has been studied by using a three dimensional hybrid simulation code, treating the electrons as a massless, charge-neutralizing fluid, whereas a completely kinetic approach is used to cover ion dynamics. The model includes two different electron populations, taking into account the significantly different temperatures of the magnetospheric and ionospheric electrons. The simulation code contains a sophisticated ionosphere model with a production rate depending on the altitude above the surface as well as on the solar zenith angle. The ionosphere is assumed to be generated by solar ultraviolet radiation. A curvilinear grid has been used to match the spherical geometry of the planetary obstacle. Several different scenarios have been taken into consideration, the purpose being to identify the general characteristics of the interaction region as a function of Saturnian local time.

When Saturn's magnetosphere is compressed due to high solar wind dynamic pressure, Titan's orbit might be loacted in the magnetosheath or even in the supersonic solar wind. Titan's interaction with a supersonic plasma flow exhibits many similarities to the Martian plasma environment: a bow shock arises in front of the obstacle, and the ionospheric nitrogen ions are clearly separated from the ambient plasma flow by means of an ion composition boundary. The convective electric field gives rise to a significant asymmetry in the structure of the interaction region. In the $E^{-}$hemisphere, this field points inward the ionospheric tail, preventing the ions from entering this hemisphere. The resulting electron pressure gradient, on the other hand, forbids the ambient plasma to mix with the ionospheric ions. In the $E^{+}$ hemisphere, the convective electric field points away from the obstacle, resulting in the formation of an extended pickup region. The general results for the supersonic case feature a strong analogy to hybrid simulation studies of the Martian plasma environment conducted by Bößwetter et al. (2004). When Titan is located inside Saturn's magnetosphere at 18:00 local time, a strong magnetic draping pattern as well as a pronounced pile-up region arise in the vicinity of the obstacle. An extended pick-up region is formed in the downstream region, again being highly asymmetric with respect to the direction of the convective electric field. However, since the density jump at the outer flank of the ionospheric tail is not as pronounced as in the supersonic scenario, the pressure gradient is no longer strong enough to prevent the ambient magnetospheric plasma flow from mixing with the ionospheric nitrogen ions. The general features of the interaction region in the 06:00 LT scenario do not differ significantly from the situation at 18:00 LT, except for the nitrogen density in the wake being around a factor 4 higher.

When Titan is located in Saturn's wake region, the upstream plasma flow is sub-Alfvénic, subsonic and submagnetosonic. In this situation, the magnetic draping pattern is significantly less pronounced than in the other scenarios 
under consideration. The peak field strength that is reached in the lobes as well as in the pile-up region at Titan's ramside is only about a factor 2 higher than the undisturbed background magnetic field. The magnetic field strength in the region between the two lobes is comparable to the undisturbed field, whereas it almost vanishes in both the 06:00 LT and the 18:00 LT scenario. In the 00:00 LT run, the interaction gives rise to an asymmetric ionospheric tail, even though the asymmetries are not as pronounced as in the other simulation runs. Because the convective electric field dragging the ionospheric particles away from Titan is weaker than in the 06:00 LT and the 18:00 LT scenario, the ionospheric tail possesses an extension of only $5 R_{T}$ in $E^{+}$direction. The magnetospheric ion density in the vicinity of Titan remains almost uninfluenced by the presence of the obstacle.

In order to improve the existing plasma model for Titan, incorporating other ionization sources, such as electron impact, will be necessary. Besides, the effects arising from a multi-component ionosphere will have to be taken into consideration. Including at least a rough approximation to the particle impact ionization processes, distinguishing between ramside and wakeside, will be the first step of these improvements. Moreover, future simulation scenarios will be designed to study the situation during specific Cassini flybys. One important aspect that we will analyze is the question whether the kinetic treatment of the ions results in the generation of electromagnetic ULF waves and what role these waves play in the overall nature of the interaction.

Acknowledgements. This work has been supported by the Deutsche Forschungsgemeinschaft through the grants MO 539/13-1 and MO 539/ 15-1. Besides, the authors thank J. Schuele from the Institute for Scientific Computing, TU Braunschweig, for numerous valuable discussions concerning the parallelization of the 3-D simulation code.

Topical Editor I. A. Daglis thanks N. Omidi and P. Israelevich for their help in evaluating this paper.

\section{References}

Backes, H., Neubauer, F. M., Dougherty, M. K., Achilleos, N., André, N., Arridge, C. S., Bertucci, C., Jones, G. H., Khurana, K. K., Russell, C. T., and Wennmacher, A.: Titan's Magnetic Field Signature During the First Cassini Encounter, Science, 308, 992-995, 2005.

Bagdonat, T.: Hybrid Simulation of Weak Comets, Ph.D. thesis, Technische Universität Braunschweig, 2004.

Bagdonat, T. and Motschmann, U.: 3D hybrid simulation of solar wind interaction with comets, in: Space Plasma Simulation Proceedings of the Sixth International School/ Symposium ISSS6, edited by: Büchner, J., Dum, C., and Scholer, M., 80-83, 2001.

Bagdonat, T. and Motschmann, U.: 3D Hybrid Simulation Code Using Curvilinear Coordinates, J. of Computational Physics, 183, 470-485, 2002a.

Bagdonat, T. and Motschmann, U.: From a weak to a strong comet - 3D global hybrid simulation studies, Earth, Moon and Planets, 90, 305-321, 2002b.
Bauer, S. J.: Physics of Planetary Ionospheres, vol. 6 of Physics and Chemistry in Space, Springer-Verlag, Berlin, Heidelberg, New York, 1973.

Bauer, S. J. and Lammer, H.: Planetary Aeronomy: Atmosphere Environments in Planetary Systems, Springer-Verlag, Berlin, Heidelberg, New York, 2004.

Baumjohann, W. and Treumann, R. A.: Basic space plasma physics, Imperial College Press, London, 1999.

Bößwetter, A., Bagdonat, T., Motschmann, U., and Sauer, K.: Plasma boundaries at Mars: a 3-D simulation study, Ann. Geophys., 22, 4363-4379, 2004.

Brecht, S., Luhmann, J. G., and Larson, D. J.: Simulation of the Saturnian magnetospheric interaction with Titan, J. Geophys. Res., 105, 13 119-13 130, 2000.

Cravens, T. E., Lindgren, C. J., and Ledvina, S. A.: A twodimensional multifluid MHD model of Titan's plasma environment, Planet. Space Sci., 46, 1193-1205, 1998.

Gurnett, D. A., Scarf, F. L., and Kurth, W. S.: The Structure of Titan's Wake From Plasma Wave Observations, J. Geophys. Res., 87, 1395-1403, 1982.

Hartle, R. E., Sittler, E. C., Oglivie, K. W., Scudder, J. D., Lazarus, A. J., and Atreya, S. K.: Titan's Ion Exosphere Observed from Voyager 1, J. Geophys. Res., 87, 1383-1394, 1982.

Kabin, K., Gombosi, T. I., DeZeeuw, D. L., Powell, K. G., and Israelevich, P. L.: Interaction of the Saturnian magnetosphere with Titan: Results of a three-dimensional MHD simulation, J. Geophys. Res., 104, 2451-2458, 1999.

Kabin, K., Gombosi, T. I., DeZeeuw, T. E., and Powell, K. G.: Interaction of Mercury with the Solar Wind, Icarus, 143, 397-406, 2000a.

Kabin, K., Israelevich, P. L., Ershkovich, A. I., Neubauer, F. M., Gombosi, T. I., DeZeeuw, D. L., and Powell, K. G.: Titan's magnetic wake: Atmospheric or magnetospheric interaction, J. Geophys. Res., 105, 10761-10770, 2000b.

Kallio, E., Sillanpää, I., and Janhunen, P.: Titan in subsonic and supersonic flow, Geophysical Research Letters, 31, L15 701/1L15 703/4, 2004.

Keller, C., Cravens, T., and Gan, L.: One-dimensional multispecies magnetohydrodynamic model of the ramside ionosphere of Titan, J. Geophys. Res., 99, 6511-6525, 1994.

Keller, C. N. and Cravens, T. E.: One-dimensional multispecies hydrodynamic models of the wakeside ionosphere of Titan, J. Geophys. Res., 99, 6527-6536, 1994.

Kivelson, M. G. and Russell, C. T.: The Interaction of Flowing Plasmas With Planetary Ionospheres: A Titan-Venus Comparison, J. Geophys. Res., 88, 49-57, 1983.

Kopp, A. and Ip, W.-H.: Asymmetric mass loading effects at Titans ionosphere, J. Geophys. Res., 106, 8323-8332, 2001.

Ledvina, S. A. and Cravens, T. E.: A three-dimensional MHD model of plasma flow around Titan, Planet. Space Sci., 46, 11751191, 1998.

Ledvina, S. A., Cravens, T. E., Salman, A., and Kecskemety, K.: Ion trajectories in Saturn's magnetosphere near Titan, Adv. Space Res., 26, 1691-1695, 2000.

Ledvina, S. A., Luhmann, J. G., Brecht, S. H., and Cravens, T. E.: Titan's induced magnetosphere, Adv. Space Res., 33, 20922102, 2004a.

Ledvina, S. A., Luhmann, J. G., and Cravens, T. E.: Ambient ion distributions in Saturn's magnetosphere near Titan during a non- 
Voyager type interaction, Adv. Space Res., 33, 221-226, $2004 \mathrm{~b}$. Ledvina, S. A., Cravens, T. E., and Kecskemety, K.: Ion distributions in Saturn's magnetosphere near Titan, J. Geophys. Res., 110, 1-16, 2005.

Luhmann, J. G.: Titan's ion exosphere wake: A natural ion mass spectrometer?, J. Geophys. Res., 101, 29387-29393, 1996.

Luhmann, J. G., Russell, C. T., Schwingenschuh, K., and Yeroshenko, Y.: A comparison of induced magnetotails of planetary bodies: Venus, Mars and Titan, J. Geophys. Res., 96, 11 199-11 208, 1991.

Ma, Y., Nagy, A., Cravens, T., Sokolov, I., Clark, J., and Hansen, K.: 3-D global MHD prediction for the first close flyby of Titan by Cassini, Geophys. Res. Lett., 31, L22083, 2004.

Nagy, A., Liu, Y., Hansen, K., Kabin, K., Gombosi, T., Combi, M., and DeZeeuw, D.: The interaction between the magnetosphere of Saturn and Titan's ionosphere, J. Geophys. Res., 106, 61516160, 2001.

Nagy, A. F. and Cravens, T. E.: Titan's ionosphere: A review, Planet. Space Sci., 46, 1149-1155, 1998.
Ness, N., Acuña, M., Behannon, K., and Neubauer, F.: The induced magnetosphere of Titan, J. Geophys. Res., 87, 1369-1381, 1982.

Roboz, A. and Nagy, A. F.: The energetics of Titan's ionosphere, J. Geophys. Res., 99, 2087-2093, 1994.

Saur, J., Mauk, B. H., Kassner, A., and Neubauer, F. M.: A model for the azimuthal plasma velocity in Saturn's magnetosphere, J. Geophys. Res., 109, 1-8, 2004.

Schardt, A. W., Behannon, K. W., Lepping, R. P., Carbary, J. F., Eviatar, A., and Siscoe, G. L.: The outer magnetosphere, in: Saturn, edited by: Gehrels, T. and Matthews, M. S., University of Arizona Press, Tucson, 416-459, 1984.

Verigin, M. I., Gringauz, K. I., and Ness, N. F.: Comparison of induced magnetospheres at Venus and Titan, J. Geophys. Res., 89, 5461-5470, 1984.

Wahlund, J.-E., Boström, R., Gustafsson, G., A., G. D., Kurth, W. S., Pedersen, A., Averkamp, T. F., Hospodarsky, G. B., Persoon, A. M., Canu, P., Neubauer, F. M., Dougherty, M. K., Eriksson, A. I., Morooka, M. W., Gill, R., André, M., Eliasson, L., and Mueller-Wodarg, I.: Cassini Measurements of Cold Plasma in the Ionosphere of Titan, Science, 308, 986-989, 2005.

Wolf, D. A. and Neubauer, F. M.: Titan's Highly Variable Plasma Environment, J. Geophys. Res., 87, 881-885, 1982. 